\title{
Dynamic Pricing of Ride-Hailing Platforms considering Service Quality and Supply Capacity under Demand Fluctuation
}

\author{
Zhongmiao Sun (iD, Qi Xu (D), and Baoli Shi \\ Glorious Sun School of Business and Management, Donghua University, 200051 Shanghai, China \\ Correspondence should be addressed to Qi Xu; xuqi@dhu.edu.cn
}

Received 7 April 2020; Accepted 28 May 2020; Published 16 July 2020

Academic Editor: Alexander Paz

Copyright (c) 2020 Zhongmiao Sun et al. This is an open access article distributed under the Creative Commons Attribution License, which permits unrestricted use, distribution, and reproduction in any medium, provided the original work is properly cited.

\begin{abstract}
Increasing attention is being paid to the pricing decisions of ride-hailing platforms. These platforms usually face market demand fluctuation and reflect supply and demand imbalances. Unlike existing studies, we focus on the optimal dynamic pricing of the platforms under imbalance between supply and demand caused by market fluctuation. Dynamic models are constructed based on the state change of supply and demand by using optimal control theory, with the aim of maximizing the platform's total profit. We obtain the optimal trajectories of price, supply, and demand under three ride demand situations. The effects of some key parameters on pricing decisions, such as coefficient of demand fluctuation, service quality, and fixed commission rate, are examined. We find the optimal dynamic price can improve the match of supply-demand in ride-hailing market and enhance the revenue of platform.
\end{abstract}

\section{Introduction}

As an innovation of the mobile Internet era, ride-hailing platforms (e.g., Uber, Lyft, and DiDi) have played a significant role in improving vehicle use efficiency, increasing transportation service supply, facilitating taxiing, and promoting employment. As such, ride-hailing platforms have been rapidly popularized and have subverted the traditional taxi market, completing millions of trips every day (Ma et al. [1]). By July 2018, Uber had completed over 10 billion ridehailing transactions globally and was active in over 80 countries and 700 cities (Uber [2]). The total market value of the global ride-hailing industry is projected to grow to $\$ 285$ billion by 2030 (MarketWatch [3]), which will bring huge economic benefits to ride-hailing platforms. Ride-hailing platforms rely on freelance drivers who decide when and for how long to work ( $\mathrm{Hu}$ and Zhou [4]). The platforms use mobile Internet to integrate the online and offline functions, match the demand of riders with the supply of drivers, reduce the empty load rate of drivers, save the cost of communication between drivers and riders, and improve the convenience and efficiency of travel (Cramer and Krueger
[5]). Compared with public transport, the benefits of ridehailing are convenience, time-saving, and comfort, which has won the favor of some ride-hailing passengers. In addition, when the weather is bad, time is tight, and travel location is remote, users can conveniently use a mobile phone platform to access a ride-hailing service.

However, since 2018, the supply of vehicles in China has been insufficient, and there has been difficulty in finding a ride-hailing service in some areas and during certain periods (Xinhua news [6]). The reason for the difficulty is mainly due to fluctuations in demand and the mismatch between supply and demand. Demand during the peak period increases rapidly, and supply cannot keep up; however, during the normal period, supply is excessive (Hu and Zhou [4]). For example, in big cities such as Beijing and Shanghai, queuing is necessary during the morning and evening peak hours and even more so during holidays. The ride-hailing platform's application usually shows that there are more than a dozen people in the queue with an average wait of 20 minutes or that the driver is far away, etc (Xinhua news [6]). To solve these difficulties, some ride-hailing platforms try to dynamically adjust the price to match the supply and demand 
(Yan et al. [7]). For example, DiDi's Spring Festival service fee and the daily price adjustment mechanism for the morning peak are based on supply and demand. Passengers willing to pay higher service fees are sent to motivated drivers who give up their holiday break. This arrangement is convenient for the passengers but also gives the drivers a reasonable and legitimate return; it is a win-win scenario (Cachon et al. [8]).

Compared with traditional taxi companies, ride-hailing platforms have their own unique characteristics. For example, ride-hailing passengers can quickly obtain ride information (e.g., the waiting time, the number of people in line, and even the number of drivers around them) and coordinate with nearby drivers in real time, whereas traditional taxi passengers usually wait at the side of the road or book in advance. In addition, the drivers of ride-hailing platforms are relatively free to choose when and where they work. The fluctuation of ride-hailing demand based on specific reasons was related to more people wanting to use the service (e.g., time of day, price, and service quality), and the supply of services usually be influenced by factors such whether there are drivers available, geographic areas, commissions etc. Due to the influence of weather factors (e.g., sunny, rainy, and snowy weather) and special occasions (e.g., after a concert), there can be a mismatch in supply and demand (Hall et al. [9]; Cachon et al. [8]; Yan et al. [7]; Afèche et al. [10]; Hu and Zhou [4]).

We are motivated by the dynamic pricing problems that arise when ride-hailing platforms face market demand fluctuation and supply-demand mismatch. Although there have been studies on pricing strategies, many authors (e.g., Feng et al. [11]; Yan et al. [7]; Hu and Zhou [12]; Sun et al. [13]; Bimpikis et al. [14]) have not designed their models to take into account the characteristic of demand fluctuation over time. They also do not consider the supply function, which is sensitive to dynamic wages, in relation to time. When rider demand increases but there is not enough supply to meet the demand (Cachon et al. [8]), platforms implement "demand rationing" by delaying the service, extending the rider wait time, or even losing the rider bookings. The difficulty in hailing a car in some regions and during certain periods affects the consumers' travel experience. When rider demand decreases, supply can exceed the demand (Cachon et al. [8]). In such cases, the platform resorts to capacity rationing, that is, to idle available transportation capacity. Therefore, to maximize the profits, it is important to determine the platforms' optimal pricing under market environments of unbalanced supply and demand caused by fluctuations in rider demand and thus reduce the rider booking delay and underutilized transportation capacity.

This study considers a ride-hailing platform in the market. The platform crowdsources drivers who have idle car resources and provides shared transport for riders. We incorporate the rider demand fluctuation into the common demand function (Desiraju and Moorthy [15]) and use the coefficient of demand fluctuation to describe different demand conditions. Unlike the previous studies (e.g., Cachon et al. [8]; Hu and Zhou [4]; Liu et al. [16]), we assume rider demand is an exponential function of time and is sensitive to price and service quality. This means rider demand changes dynamically over time.

As a service enterprise, the ride-hailing platform can provide ride supply-demand matching services that can be reflected by service quality. The service quality usually refers to passenger satisfaction with ride-hailing services, such as passengers' ride efficiency, platforms' supervision, and use experience of APP. In reality, the passengers are concerned not only with the price but also with the service quality. However, service quality is closely related to service cost, which affects the platform's pricing decisions. Therefore, it is necessary to study the impact of service quality on platform pricing decisions, transaction volumes, and profits. Furthermore, the fixed commission rate is the percentage of the fare that the platform pays to the drivers after they complete each ride (Cachon et al. [8] and Hu and Zhou [4]). It directly affects not only the platform's revenue from each transaction but also the drivers' participation through their income. Therefore, we take into account the service quality and the fixed commission rate of the ride-hailing platforms, the opportunity cost when supply exceeds demand, and the booking delay cost when supply fails to meet demand in time. We thus study three different demand situations: decrease in demand, increase in demand, and stable demand. Optimal control theory has been used in the literature on dynamic pricing (e.g., $\mathrm{Lu}$ et al. [17]; Herbon and Khmelnitsky [18]; and Feng et al. [19]). Thus, we use optimal control theory to construct models and study the dynamic pricing of ride-hailing platforms with different market demand situations in a finite service time $[0, T]$. An analysis of key parameters reveals further insights into the effect of the coefficient of demand fluctuation, service quality, and fixed commission rate on the platform's pricing decisions. We find that, although the peak congestion period can be reduced by reducing service quality and/or increasing the fixed commission rate, the reduction in the service quality may not be desirable due to the loss of peak demand.

We investigate the impact of key parameters on the total transaction volume and the total profit in a finite service time, in which the total profit is a concave function of the fixed commission rate and service quality. To the best of our knowledge, few studies have investigated dynamic pricing according to the characteristics of demand fluctuation in the actual operation of ride-hailing platforms. Our study is the first to assume demand is an exponential function of time and to use optimal control theory to examine the pricing problem of ride-hailing platforms. We address the following research questions:

(1) How do the ride-hailing platforms develop effective dynamic pricing strategies in the face of demandsupply imbalances under different market demand situations (decreased demand, increased demand, and stable demand), and how do the trajectories of the platforms' supply rate and demand rate change under the influence of optimal dynamic prices?

(2) If the opportunity cost is not considered, how does the platform adjust its optimal pricing strategy if market demand decreases? If a ceiling price 
constraint is considered, how do the optimal pricing strategies change if market demand increases?

(3) How do the coefficient of demand fluctuation, service quality, and fixed commission rate affect pricing decisions, transaction volumes, and profits under different market demand situations?

\section{Literature Review}

2.1. Two-Sided Markets. There are a number of studies on two-sided markets in various industries, such as the credit card industry (Rochet and Tirole [20, 21]; Armstrong [22]), the telecoms market (Waverman [23]), online gaming (Parker and Van Alstyne [24]), e-commerce (Gaudeul and Jullien [25]), media (Anderson and Gabszewicz [26]; Kind and Stähler [27]), and transportation (Furuhata et al. [28]; Wang et al. [29]; Djavadian and Chow [30]). Rochet and Tirole [20], Gaudeul and Jullien [31], and Armstrong [22] have done pioneering research on two-sided markets. Rochet and Tirole [21] defined two-sided markets: "A market is two-sided if the platform can affect the volume of transactions by changing the price share between two groups." Most of the literature considers network externalities in pricing strategies (e.g., Rochet and Tirole [20, 21]; Parker and Van Alstyne [24]; Wang et al. [29]; Djavadian and Chow [30]). Some studies focus on competition (e.g., Rochet and Tirole [20]; Armstrong [22]; Kind and Stähler [27]).

However, unlike most two-sided markets, ride-hailing platforms have pricing power and need to match the supply with the demand in a timely manner. In our study, rather than focusing on equilibrium and the impact of network externalities, we examine the dynamic setting in which a ride-hailing platform sets price and wage over time under different market demand situations. Specifically, we refer to the description by Hu and Zhou [4] in which the platform's actual transaction volume at time $t$ is the minimum of rider demand and supply capacity. That is, if in a period, there are $D$ riders and $S$ drivers at time $t$ in a neighborhood, the actual transaction volume at time $t$ is close to $\min \{S, D\}$.

2.2. Pricing Problems. Optimal pricing problems have been extensively studied in the revenue management and pricing literature (Kolisch and Zatta [32]). Most revenue management studies usually consider a fixed supply side and pricesensitive demand (Petruzzi and Dada [33]). In contrast, we consider a time-varying supply side that is sensitive to the wage offered by the ride-hailing platform.

Our study is related to the dynamic pricing in operation management. Over the past two decades, dynamic pricing has attracted considerable attention from both industry and academia (Cao et al. [34]). Gallego and van Ryzin [35] first connected dynamic pricing with revenue management and applied the intensity control theory system to the dynamic pricing of perishable goods and later extended their research to multiproduct situations (Gallego and van Ryzin [36]). Smith and Achabal [37] posited that the sales rate is related to seasonal factors of price and the level of residual inventory; they established an optimized model based on the pricing problem of retail products at the end of the quarter. Some authors consider the consumer strategic behavior (e.g., Besanko and Winston [38]; Anderson and Wilson [39]; Aviv and Pazgal [40]; Levin et al. [41]). In the recent years, there have been many studies on dynamic pricing (e.g., $\mathrm{Hu}$ et al. [42]; Varella et al. [43]; Ajorlou et al. [44]; Feng et al. [19]; Chen and Chen [45]), especially regarding perishable items.

$\mathrm{Lu}$ et al. [17] introduced a perishable item inventory system with limited replenishment capacity and achieved an optimal joint dynamic pricing and replenishment strategy by solving the optimal problem using Pontryagin's maximum principle. Herbon and Khmelnitsky [18] used optimal control theory to study the optimal dynamic pricing and optimal replenishment strategies of storable perishable items. Feng [19] studied the dynamic optimization model for the maximization of total profit per unit time of perishable products with dynamic pricing and quality investment; they determined an optimal joint dynamic pricing, quality investment, and replenishment strategy based on Pontryagin's maximum principle. Related studies include Chen et al.'s [46] and Sato's study [47]. These authors used optimal control theory (Lu et al. [17]; Herbon and Khmelnitsky [18]; Feng [19]) to construct an equation of state change and obtain an optimal dynamic price solution according to Pontryagin's maximum principle. We construct an equation of state change based on supply and demand and study the ride-hailing platforms' dynamic pricing of ride-hailing platforms using optimal control theory to maximize the total profit in a period.

2.3. Ride-Hailing Platforms. The service operation of ridehailing platforms, based on the concept of "shared transport," has increasingly attracted the scholars to do research in the field of operation management. At present, research on the service operation of ride-hailing platforms focuses on the pricing strategy and on the matching supply with demand. To examine platform pricing strategy, Cachon et al. [8] designed five contracts by adjusting prices and commissions of ride-hailing platforms and found that the optimal contract substantially increases the platform's profit, relative to contracts with a fixed price or fixed wage (or both), and although surge pricing is not optimal, it generally achieves optimal profit. Zha et al. [48] studied the peak pricing effect on ride-hailing platforms under different assumptions of labor supply behavior. Yan et al. [7] used data from Uber to show that, by jointly optimizing the dynamic pricing and dynamic waiting, price variability can be mitigated while increasing the capacity utilization, trip throughput, and welfare. Guda and Subramanian [49] considered the strategic interactions among ride-hailing platform drivers when deciding to move across regions and found that even in areas where driver supply exceeds demand, peak pricing can be profitable. Bimpikis et al. [14] explored the spatial price discrimination in the context of a ride-hailing platform that serves a network of locations. Sun et al. [13] were the first to take into account both ride details 
and driver locations and assumed that drivers and customers maximize the utility to determine the optimal pricing strategy for online ride-hailing platforms. Liu et al. [16] studied the pricing decisions of a profit-maximizing platform by considering the provider's threshold participating quantity, value-added service (VAS), and matching ability. Wu et al. [50] considered spatial differentiation and network externality in the pricing mechanism of an online car-hailing platform and analyzed the game competition among platform users and the impact on the platform's optimal pricing. Although the literature has demonstrated and studied the pricing strategies of ride-hailing platforms at peak demand, these studies have not incorporated the characteristics of demand fluctuation and uncertain supply into the models nor have they explored how service quality and the platform's fixed commission rates affect the pricing decisions, transaction volume, and profit.

On matching the supply with demand, many researchers modeled the matching process between drivers and customers as an unobservable queue. The arrival of passengers is regarded as a Poisson process, and drivers are regarded as servers in the queuing system (Bai et al. [51]; Taylor [52]; Hu and Zhou [4]), with queuing theory used to study the supply and demand matching. Feng et al. [11] compared the efficiency of online supply and demand matching with the average waiting time of traditional taxi hailing. Hu and Zhou [12] considered an intermediary's problem of dynamically matching demand and supply of heterogeneous types in a periodic review. In research on other aspects of ride-hailing platforms, Ma et al. [1] studied the optimal scheduling that would enable drivers to choose to accept the platform's scheduling rather than drive to another area or wait for higher prices. Afèche et al. [10] found that drivers make strategic decisions on whether and where to provide services according to soaring price and income differences.

2.4. Academic Contribution. Our paper differs from the above studies in two aspects. First, although it is common to study pricing strategies in different industries using optimal control theory, few researchers have used this method to study the pricing strategies of ride-hailing platforms. We introduce optimal control theory to study the pricing problems of ride-hailing platforms. Thus, we extend the existing research methods to ride-hailing platforms. Second, we consider the characteristics of demand fluctuation into the models, in view of the practices of such platforms as $\mathrm{DiDi}$ and Uber that face the ride-hailing demand vary dynamically with time at different periods. The optimal dynamic price trajectory of ride-hailing platforms are investigated, and we find out the key time points that affect the platform optimization pricing under different circumstances, so as to make optimal pricing decisions for different ride time periods be more practical guiding. In addition, we examine the impacts of platform service quality, fixed commission rate, and market demand fluctuation on the pricing decisions, transaction volume, and profit under different market situations.

\section{Problem Description and Model Assumptions}

Ride-hailing platforms match riders and drivers by coordinating the supply and demand of ride services. Drivers can freely choose to receive bookings and complete offline transportation services. Once the ride is complete, riders usually pay fares through third-party payment platforms (e.g., Alipay or WeChat) on their mobile phones, and the drivers receive a corresponding commission from the ridehailing platform. The operational process of ride-hailing platforms is shown in Figure 1.

We consider a ride-hailing market consisting of a ridehailing platform, a number of drivers, and a number of riders. In a finite service time $[0, T]$, the platform's demand rate is $D(t)$ and the platform's supply rate is $S(t)$. The platform charges the riders price $P(t)$ based on the supply and demand situation at time $t$. The platform pays commission $W(t)$ to the drivers who use their personal vehicles and provide the transportation service. When the supply rate is higher than the demand rate, the platform bears unit opportunity cost $c$. When the supply rate cannot meet the demand rate, the platform bears unit booking delay cost $h$. Note that opportunity cost $c$ or booking delay cost $h$ are incurred per time unit. Such a ride-hailing platform is illustrated in Figure 2.

The assumptions used to formulate the problem are as follows (the list of notations is in Table 1):

(1) It is assumed that the supply and demand at time 0 are in balance under the dynamic pricing policy, that is, $D(0)=S(0)$.

(2) In the Uber setting, the "surge multiplier" multiplied by the base fare (as a whole, corresponding to the price in our model), together with the travel time and distance, determines the trip fare. For simplicity, our model assumes away the spatial dimension (e.g., Cachon et al. [8]; Hu and Zhou [4]).

(3) Referring to Liu et al. [16], the demand rate for ridehailing platforms is sensitive to price and service quality and is assumed to be a linear function of the price:

$$
D(P, t)=\alpha(t)-\beta P(t)+\gamma q,
$$

where $\alpha(t)$ represents the initial demand of the market in a certain region, $\beta>0$ indicates the price sensitivity factor, and $\gamma>0$ is the service sensitivity factor. In addition, $D(P, t) \geqq 0$ at the ride service time $[0, T]$; otherwise, this model would be uninteresting.

(4) We assume the following supply rate function:

$$
S(W, t)=s \cdot(W(t)-\varepsilon),
$$

where $W(t)$ represents the commission for drivers, $W(t)=r \cdot P(t)$ (Cachon et al. [8]; Hu and Zhou [4]), $\varepsilon$ is the minimum participation cost per time unit of a driver, which indicates a driver is willing to accept a ride if the commission $W(t)$ exceeds $\varepsilon$ (Sun et al. [13]) 


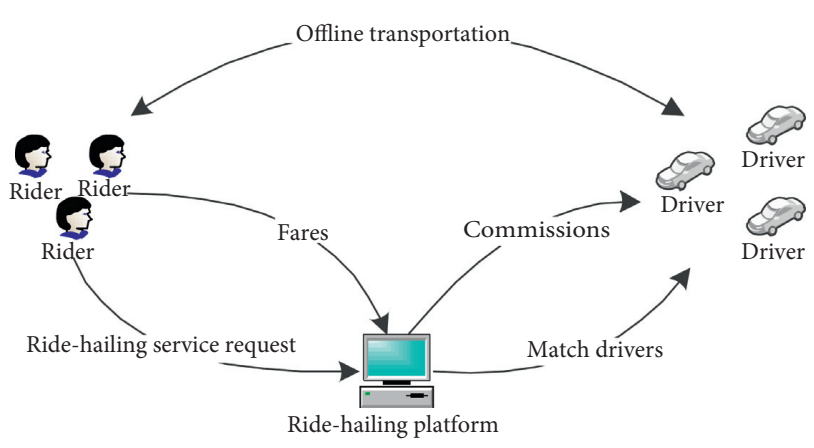

FIgURE 1: Operational process of ride-hailing platforms.

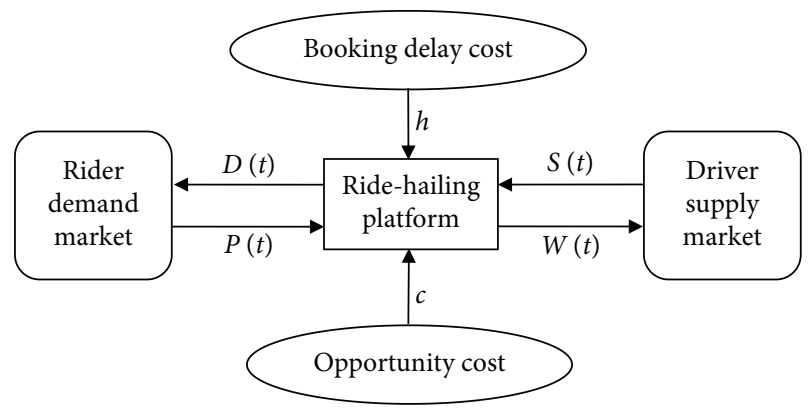

FIgURE 2: Model of a ride-hailing platform.

TABLE 1: Notations.

\begin{tabular}{|c|c|}
\hline \multicolumn{2}{|l|}{ Index } \\
\hline$v$ & The situation in decreasing market demand \\
\hline$u$ & The situation in increasing market demand \\
\hline$b$ & The situation in stable market demand \\
\hline$t$ & Time, $t \in[0, T]$ \\
\hline \multicolumn{2}{|c|}{ Parameters } \\
\hline$a$ & The coefficient of demand fluctuation \\
\hline$\alpha$ & The size of rider demand market at $t=0$ or $a=0$ \\
\hline$\beta$ & The price sensitivity factor \\
\hline$\gamma$ & The service sensitivity factor \\
\hline$s$ & The wage sensitivity factor \\
\hline$r$ & The fixed commission rate, $0<r<1$ \\
\hline$\eta$ & The service cost parameter \\
\hline$q$ & Service quality \\
\hline$c$ & The unit opportunity cost \\
\hline$h$ & The unit booking delay cost \\
\hline$\varepsilon$ & Minimum participation cost per time unit of a driver \\
\hline $\bar{P}$ & The ceiling price \\
\hline$P(t)$ & The ride-hailing service price \\
\hline$W(t)$ & The commission (wage) for drivers \\
\hline$C(t)$ & The unit cost function \\
\hline$\alpha(t)$ & The initial demand of the market at time $t$ \\
\hline$D(P, t)$ & The demand rate function \\
\hline$S(W, t)$ & The supply rate function \\
\hline$v(t)$ & The excess supply capacity of the platform \\
\hline$u(t)$ & The quantity of delayed bookings of the platform \\
\hline$\psi(P, t)$ & The total transaction volume of the platform \\
\hline$\pi(P, t)$ & The total profit of the platform \\
\hline
\end{tabular}

(i.e., $W(t)>\varepsilon$ ), and $s$ is the wage sensitivity factor, which reflects the sensitivity of supply to changes in commission ( $\mathrm{Hu}$ and Zhou [4]).
According to the demand rate and supply rate models above, the platform's total transaction volume is as follows:

$$
\psi(P, t)=\int_{0}^{T} \min (D(P, t), S(W, t)) \mathrm{d} t .
$$

(5) The ride-hailing platform's unit cost function is as follows:

$$
C(t)=W(t)+\eta q^{2}
$$

where $\eta>0$ is the platform's service cost parameter, $q$ is the platform's service quality, and $\eta q^{2}$ has been widely used (e.g., Moorthy [53]; Motta [54]; Ha et al. [55]).

(6) During the ride service time $[0, T]$, when $D(P, t) \leqq$ $S(W, t)$, the ride demand decreases and the supply capacity of the platform is larger than the demand. Let $v(t)$ represent the platform's excess supply capacity and $v(0)=0$. When $D(P, t) \geqq S(W, t)$, the ride demand increases and thus some ride requirements cannot be met in time. Let $u(t)$ represent the quantity of delayed bookings and $u(0)=0$.

(7) To describe the effect of market demand fluctuation, we assume that the initial demand of the market in a certain region over time is as follows:

$$
\alpha(t)=\alpha e^{-a t},
$$

where $\alpha>0$ is the size of the rider demand market at $t=0$ or $a=0$. Referring to Maihami and Kamalabadi [56] and Ghoreishi et al. [57], $\alpha e^{-a t}$ describes the fluctuation for the initial ride demand in a certain region (i.e., decreasing, increasing, and stable). The coefficient of demand fluctuation $a$ represents a decreasing market demand if $a>0$, as shown in Figure 3, which is consistent with the real scenario of decreasing market demand (e.g., when the demand decreases after rush hour). If $a<0$, the market demand increases, as shown in Figure 4, which is consistent with the real scenario of increasing market demand (e.g., when the demand increases during rush hour, during rainy/snowy weather, or at the end of a large community event). If $a=0$, it represents the stable market demand, which is between decreasing market demand and increasing market demand.

\section{Model Analysis}

In Sections 4.1, 4.2, and 4.3, we present the optimal pricing strategies in a finite service time $[0, T]$ under three ride market situations: decreasing demand, increasing demand, and stable demand. Based on three optimal pricing strategies, we obtain the platform's supply rate, demand rate, total transaction volume, and total profit.

4.1. Optimal Pricing Strategies with Decreasing Market Demand $(a>0)$. Decreasing market demand indicates that people's demand for transportation is decreasing within a 


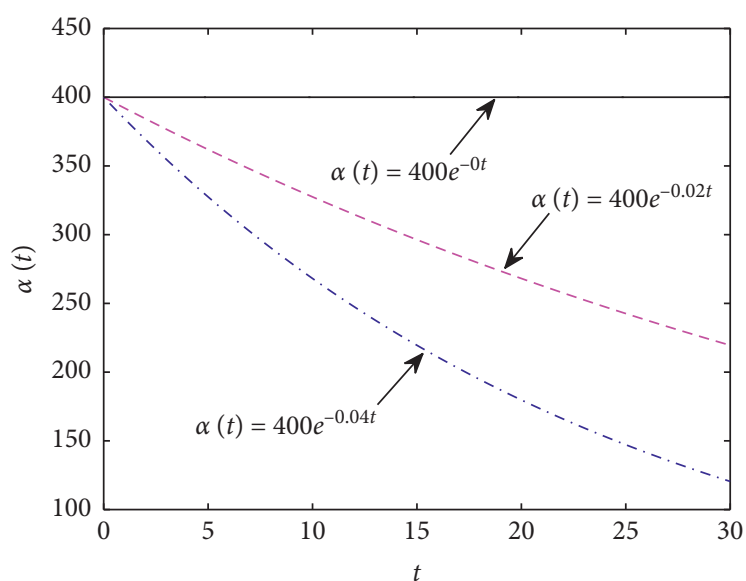

FIgURE 3: Decreasing market demand.

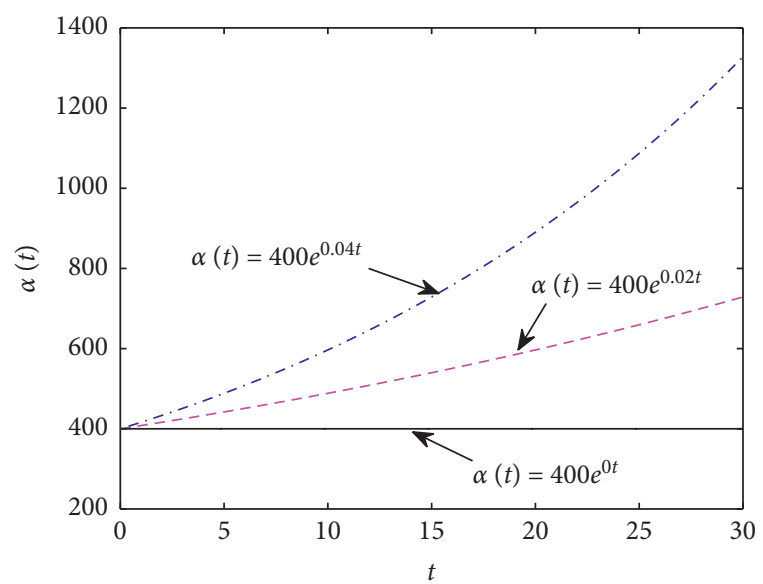

FIGURE 4: Increasing market demand.

period $[0, T]$, such as during weekday nonrush hours (see Figure 3). Therefore, the balance of the supply and demand of the ride-hailing platform at time $t=0$ will be broken. Excess supply capacity $(v(t) \geqq 0)$, or capacity rationing, occurs during this period (Cachon et al. [8]). Based on the problem description and the model assumptions in Section 3 , we set price $P_{v}(t)$ of the ride-hailing service as the control variable. The optimal pricing model can be constructed with optimal control theory when the platform's supply capacity is in excess. As in Jørgensen and Kort [58], the inventory of physical products is the state variable, and the equation of state change is based on the inventory replenishment rate and the demand rate. In our setup, the state of the ride-hailing platform at time $[0, T]$ is described by the accumulated excess supply capacity rate $\bar{v}(t)$. We construct the state change equation of excess supply capacity based on the platform's supply rate $S(t)$ and demand rate $D(t)$ :

$$
\bar{v}(t)=S_{v}\left(W_{v}, t\right)-D_{v}\left(P_{v}, t\right) .
$$

The accumulated excess supply capacity at time $t$ is

$$
v(t)=v(0)+\int_{0}^{t}\left(S_{v}\left(W_{v}, \tau\right)-D_{v}\left(P_{v}, \tau\right)\right) \mathrm{d} \tau .
$$

The ride-hailing platform's objective is to find the optimal dynamic pricing and ride supply to maximize the total profit over the ride service time $[0, T]$. The objective function is given as follows:

$$
\begin{aligned}
\pi_{v}\left(P_{v}, t\right)= & \max _{P_{v}(t)} \int_{0}^{T}\left[D_{v}\left(P_{v}, t\right) \cdot P_{v}(t)-D_{v}\left(P_{v}, t\right)\right. \\
& \left.\cdot C_{v}(t)-c \cdot v(t)\right] \mathrm{d} t \\
= & \max _{P_{v}(t)} \int_{0}^{T}\left[D_{v}\left(P_{v}, t\right) \cdot\left(P_{v}(t)-W_{v}(t)-\eta q^{2}\right)\right. \\
& \left.-c\left(\int_{0}^{T} \int_{0}^{t}\left(S_{v}\left(W_{v}, \tau\right)-D_{v}\left(P_{v}, \tau\right)\right) \mathrm{d} \tau\right)\right] \mathrm{d} t \\
= & \max _{P_{v}(t)} \int_{0}^{T}\left[D_{v}\left(P_{v}, t\right) \cdot\left(P_{v}(t)-W_{v}(t)-\eta q^{2}\right)\right. \\
& \left.-c(T-t) \cdot\left(S_{v}\left(W_{v}, t\right)-D_{v}\left(P_{v}, t\right)\right)\right] \mathrm{d} t,
\end{aligned}
$$

with the following constraints:

$$
\begin{aligned}
\bar{v}(t) & =S_{v}\left(W_{v}, t\right)-D_{v}\left(P_{v}, t\right) \\
& =s \cdot\left(W_{v}(t)-\varepsilon\right)-\alpha e^{-a t}+\beta P_{v}(t)-\gamma q, \\
v(t) & \geq 0, \\
v(0) & =0, \\
a & >0 .
\end{aligned}
$$

To solve the optimization model above, the Hamilton equation satisfied by a certain value function is constructed as

$$
\begin{aligned}
H_{v} & \left(v(t), P_{v}(t), \lambda_{v}(t), t\right) \\
= & D_{v}\left(P_{v}, t\right) \cdot\left(P_{v}(t)-W_{v}(t)-\eta q^{2}\right)-c(T-t) \\
& \cdot\left(S_{v}\left(W_{v}, t\right)-D_{v}\left(P_{v}, t\right)\right) \\
& +\lambda_{v}(t) \cdot\left(s W_{v}(t)-\alpha e^{-a t}+\beta P_{v}(t)-\gamma q\right) \\
= & \left(\alpha e^{-a t}-\beta P_{v}(t)+\gamma q\right) \cdot\left(P_{v}(t)-W_{v}(t)-\eta q^{2}\right) \\
& +\left(\lambda_{v}(t)-c(T-t)\right) \\
& \cdot\left(s \cdot\left(W_{v}(t)-\varepsilon\right)-\alpha e^{-a t}+\beta P_{v}(t)-\gamma q\right),
\end{aligned}
$$

where the Lagrange multiplier $\lambda_{v}(t)$ is the costate variable associated with the state variables $S(t)$ and $D(t)$ and $\lambda_{v}(t)$ denotes the shadow price of the excess supply capacity.

Thus, we can state the solution for the scenario in which the market ride demand decreases.

Lemma 1. With decreasing market demand, the platform's profit $\pi_{v}\left(P_{v}, t\right)$ is strictly concave in service price $P_{v}(t)$, and the optimal dynamic price solution $P_{v}^{*}(t)$ can maximize the platform's profit $\pi_{v}\left(P_{v}, t\right)$.

The proof is in Appendix.

The maximum principle states that the necessary conditions for $P_{v}^{*}(t)$, with corresponding state trajectory $v^{*}(t)$, to be an optimal control are the continuous and piecewise 
differentiable function $\lambda_{v}(t)$ such that the following conditions hold. According to the necessary conditions of Pontryagin's maximum principle, we obtain the following costate equations:

$$
\begin{aligned}
\bar{v}(t) & =\frac{\partial H_{v}}{\partial \lambda_{v}}, \\
\overline{\lambda_{v}}(t) & =-\frac{\partial H_{v}}{\partial v}, \\
\frac{\partial H_{v}}{\partial P_{v}} & =0 .
\end{aligned}
$$

From (9) and (10), the optimal dynamic price and the shadow price $\lambda_{v}^{*}(t)$ can be obtained as follows:

$$
\begin{aligned}
P_{v}^{*}(t)= & \frac{\alpha}{2 \beta} e^{-a t}+\frac{c(s r+\beta)}{\beta(1-r)} t+\frac{\gamma q+s \varepsilon}{s r+\beta}-\frac{c T(s r+\beta)}{2 \beta(1-r)} \\
& -\frac{\alpha(s r-\beta) \cdot\left(1-e^{-a T}\right)}{2 \beta T a(s r+\beta)}+\frac{v(T)}{T(s r+\beta)}, \\
\lambda_{v}^{*}(t)= & c t+\frac{(1-r) \cdot(s r-\beta) \cdot\left(\alpha e^{-a T}-a T(\gamma q+s \varepsilon)-\alpha\right)}{T a(s r+\beta)^{2}} \\
& +\frac{2 \beta(1-r) \cdot v(T)}{T(s r+\beta)^{2}}-\frac{\beta \eta q^{2}}{s r+\beta} .
\end{aligned}
$$

Based on Lemma 1, the optimal pricing control problems are given in Theorem 1 .

Theorem 1. During the ride service time [0,T], when the market demand is decreasing, the optimal trajectory of the platform's dynamic price $P_{v}^{*}(t)$ is

$$
P_{v}^{*}(t)=\frac{\alpha}{2 \beta} \cdot e^{-a t}+\frac{c(s r+\beta)}{\beta(1-r)} \cdot t+\frac{\gamma q+s \varepsilon}{(s r+\beta)}-\frac{\alpha(s r-\beta)}{2 \beta(s r+\beta)} .
$$

The optimal trajectory of the shadow price $\lambda_{v}^{*}(t)$ is

$$
\begin{aligned}
\lambda_{v}^{*}(t)= & c \cdot t+\frac{(1-r) \cdot(2 \beta \alpha-(\gamma q+s \varepsilon) \cdot(s r-\beta))}{(s r+\beta)^{2}} \\
& -\frac{\left(\alpha(1-r)+\beta \eta q^{2}\right)}{s r+\beta}+c T .
\end{aligned}
$$

The optimal trajectories of the platform's supply rate $S_{v}^{*}(t)$ and demand rate $D_{v}^{*}(t)$ over time are as follows:

$$
\begin{gathered}
S_{v}^{*}(t)=\frac{s r \alpha}{2 \beta} \cdot e^{-a t}+\frac{s r c(s r+\beta)}{\beta(1-r)} \cdot t+\frac{s(r \gamma q-\beta \varepsilon)}{(s r+\beta)}-\frac{s r \alpha(s r-\beta)}{2 \beta(s r+\beta)} \\
D_{v}^{*}(t)=\frac{\alpha}{2} \cdot e^{-a t}-\frac{c(s r+\beta)}{(1-r)} \cdot t+\frac{s(r \gamma q-\beta \varepsilon)}{(s r+\beta)}+\frac{\alpha(s r-\beta)}{2(s r+\beta)} .
\end{gathered}
$$

Remark 1. In this section, we set the constraint $v(t) \geqq 0$ in (9), which represents the scenario in which the platform's demand is not higher than the supply when the market ride demand decreases in a finite service time $[0, T]$. Given the optimal solution of Theorem 1, the parameters satisfy the conditions as follows: (a) $\beta \geqq s r$; (b) if $\beta<s r$, $2 c(s r+\beta)^{2} \geq a \alpha(s r-\beta) \cdot(1-r)$.

The proof is in Appendix.

Theorem 1 indicates that the ride-hailing platform's optimal dynamic price, shadow price, supply rate, and demand rate all change dynamically with time $t$ in the ride service time $[0, T]$. Furthermore, the shadow price $\lambda_{v}^{*}(t)$ is a monotonic increasing function of time $t$, and its slope gives the unit opportunity $\cos t c$. As the shadow price represents the influence of constraint conditions on the objective function, through equation (15), we know that the change rate of the platform's excess supply capacity has an increasing impact on its profit rate with the passage of time.

Corollary 1. During the ride service time [0,T], when the market demand decreases, we conclude the following:

(a) $P_{v}^{*}(t)$ is a convex function of time $t$.

(b) Assuming $c=0$, there is a threshold $T_{t h}$. When $0 \leqq T_{t h} \leqq T, P_{v}^{*}(t)$ decreases first and then remains stable or unchanged, and when $T<T_{t h}, P_{v}^{*}(t)$ monotonically decreases.

(c) Assuming $c \neq 0$, we have a critical point $t_{1}=-(1 / a) \ln (2 c(s r+\beta) / a \alpha(1-r))$. When $0 \leqq$ $t_{1} \leqq T, P_{v}^{*}(t)$ decreases first and then increases and $P_{v}^{*}(\min )=P_{v}^{*}\left(t_{1}\right)$, and when $T<t_{1}, P_{v}^{*}(t)$ monotonically decreases.

The proof is in Appendix.

Corollary 1 shows the optimal pricing strategies when a ride-hailing platform faces decreasing market demand during the ride service time $[0, T]$. First, the platform's optimal price decreases monotonically over time. On the one hand, the platform reduces the optimal price $P_{v}^{*}(t)$ over time both to alleviate the weakening market demand and to stimulate market demand as much as possible to maximize the utilization of supply capacity. On the other hand, the platform adopts a fixed commission contract (e.g., Uber) to pay $W_{v}^{*}(t)$ for drivers, where $W_{v}^{*}(t)=r \cdot P_{v}^{*}(t)$. Thus, reducing the service price $P_{v}^{*}(t)$ means correspondingly reducing the commission $W_{v}^{*}(t)$ of drivers who join the platform to provide ride service. The platform can avoid excessive supply capacity during the period of decreasing demand to reduce the waste of idle transportation resources.

Second, with the passage of time, if the platform does not consider the opportunity cost $(c=0)$, the optimal price eventually tends to be stable or unchanged over time, which ensures basic income for the platform. In contrast, if the platform considers the opportunity cost $(c>0)$, the optimal price first decreases to a certain level $P_{v}^{*}(\min )=$ $P_{v}^{*}\left(t_{1}\right)$ and then increases over time. When the market demand decreases to an extent such that the accumulation of opportunity cost has an increasing impact on the platform's profit, the platform must raise the price to maximize profit. 
Corollary 2. During the ride service time [0, T], market demand decreases. The platform's total transaction volume $\psi_{v}^{*}$ is

$$
\begin{aligned}
\psi_{v}^{*}(t)= & \frac{\alpha\left(1-e^{-a T}\right)}{2 a}-\frac{c T^{2} \cdot(s r+\beta)}{2(1-r)} \\
& +T \cdot \frac{s(r \gamma q+\beta \varepsilon)+\alpha(s r-\beta)}{2(s r+\beta)} .
\end{aligned}
$$

The platform's total profit $\pi_{v}^{*}$ is

$$
\begin{aligned}
\pi_{v}^{*}= & \int_{p}^{T}\left[D_{v}^{*}(t) \cdot\left((1-r) \cdot P_{v}^{*}(t)-\eta q^{2}\right)\right. \\
& \left.-c \cdot \int_{0}^{t}\left(S_{v}^{*}(\tau)-D_{v}^{*}(\tau)\right) \mathrm{d} \tau\right] \mathrm{d} t .
\end{aligned}
$$

The proof is in Appendix.

4.2. Optimal Pricing Strategies with Increasing Market Demand $(a<0)$. During peak ride periods, such as during rush hour or rainy/snowy weather and at the end of large-scale community activities, the market demand for rides surges. The increasing rate of demand is shown in Figure 4. At this time, the balance of the supply and demand of the ride-hailing platform at time $t=0$ might be broken. Demand can exceed supply capacity at the ride service time $[0, T]$ (Cachon et al. $[8]$ ), and thus demand rationing can occur. Ride bookings will be delayed $(u(t) \geqq 0)$. In this situation, if the platform dynamically adjusts price according to the change in market demand, it is conducive to greater profits and better matching of supply with demand. In practice, price raising strategies are used when the platform faces high demand. Uber calls this strategy "surge pricing," and Lyft calls it "prime time."

Based on the problem description and the model assumptions in Section 3, let price $P_{u}(t)$ of the ride-hailing service be the control variable and the volume of delayed bookings be the state variable. Now, we can construct the optimal pricing model using optimal control theory (Herbon and Khmelnitsky [18]; Jørgensen and Kort [58]). First, the state change equation of the quantity of delayed bookings is constructed based on the platform's supply rate $S(t)$ and demand rate $D(t)$ :

$$
\bar{u}(t)=D_{u}\left(P_{u}, t\right)-S_{u}\left(W_{u}, t\right) .
$$

Thus, the quantity of delayed bookings at time $t$ is

$$
u(t)=u(0)+\int_{0}^{t}(D(P, \tau)-S(P, \tau)) \mathrm{d} \tau .
$$

During the ride service time $[0, T]$, the platform faces increasing market demand. As the actual supply capacity cannot meet the demand in time, the platform's actual transaction volume at time $t$ is $\min \left(D_{u}\left(P_{u}, t\right)\right.$, $\left.S_{u}\left(W_{u}, t\right)\right)=S_{u}\left(W_{u}, t\right)$. The ride-hailing platform's objective is to maximize the total profit in the ride service time [0, $T]$. The objective function is given by

$$
\begin{aligned}
\pi_{u}\left(P_{u}, t\right)= & \max _{P_{u}(t)} \int_{0}^{T}\left[S_{u}\left(W_{u}, t\right) \cdot P_{u}(t)-S_{u}\left(W_{u}, t\right) \cdot C_{u}(t)\right. \\
& -h \cdot u(t)] \mathrm{d} t \\
= & \max _{P_{u}(t)} \int_{0}^{T}\left[S_{u}\left(W_{u}, t\right) \cdot\left(P_{u}(t)-W_{u}(t)-\eta q^{2}\right)\right. \\
& \left.-h\left(\int_{0}^{t}\left(D_{u}\left(P_{u}, \tau\right)-S_{u}\left(W_{u}, \tau\right)\right) \mathrm{d} \tau\right)\right] \mathrm{d} t \\
= & \max _{P_{u}(t)} \int_{0}^{T} \int_{0}^{T}\left[S_{u}\left(W_{u}, t\right) \cdot\left(P_{u}(t)-W_{u}(t)-\eta q^{2}\right)\right. \\
& \left.-h(T-t) \cdot\left(D_{u}\left(P_{u}, t\right)-S\left(W_{u}, t\right)\right)\right] \mathrm{d} t .
\end{aligned}
$$

The constraints of (22) are as follows:

$$
\begin{aligned}
\bar{u}(t) & =D_{u}\left(P_{u}, t\right)-S_{u}\left(W_{u}, t\right) \\
& =\alpha e^{-a t}-\beta P_{u}(t)+\gamma q-s \cdot\left(W_{u}(t)-\varepsilon\right), \\
u(t) & \geq 0, \\
u(0) & =0, \\
a & <0 .
\end{aligned}
$$

We seek the platform's optimal price in the case of increasing demand. We introduce the Lagrange multiplier $\lambda_{u}(t)$ and construct the Hamilton function as follows:

$$
\begin{aligned}
H_{u} & \left(u(t), P_{u}(t), \lambda_{u}(t), t\right) \\
= & S_{u}\left(W_{u}, t\right) \cdot\left(P_{u}(t)-W_{u}(t)-\eta q^{2}\right) \\
& -h(T-t) \cdot\left(D_{u}\left(P_{u}, t\right)-S_{u}\left(W_{u}, t\right)\right) \\
& +\lambda_{u}(t) \cdot\left(\alpha e^{-a t}-\beta P_{u}(t)+\gamma q-s \cdot\left(W_{u}(t)-\varepsilon\right)\right) \\
= & \left(s W_{u}(t)\right) \cdot\left(P_{u}(t)-W_{u}(t)-\eta q^{2}\right)+\left(\lambda_{u}(t)-h(T-t)\right) . \\
& \left(\alpha e^{-a t}-\beta P_{u}(t)+\gamma q-s \cdot\left(W_{u}(t)-\varepsilon\right)\right) .
\end{aligned}
$$

Lemma 2. In increasing market demand, the platform's profit $\pi_{u}\left(P_{u}, t\right)$ is strictly convex in service price $P_{u}(t)$. There is no maximum point $P_{u}(t)$ that can maximize the platform's profit $\pi_{u}\left(P_{u}, t\right)$.

The proof is in Appendix.

Lemma 2 shows that this model cannot use Pontryagin's principle; otherwise, a price solution can be obtained to minimize the platform's profit. However, we should find the optimal dynamic price to maximize the platform's profit during the ride service time $[0, T]$. We consider two cases: (i) the platform is not constrained by the ceiling price $\left(P_{u}(t) \leqq \bar{P}, \bar{P} \longrightarrow+\infty\right)$; and (ii) the platform is constrained by the ceiling price $\left(P_{u}(t) \leqq \bar{P}, \bar{P}\right.$ is a constant), which is motivated by the reality (e.g., the government wants to avoid the malicious price increase of ride-hailing platforms in some regions, that is, regulatory constraints). Respectively, we propose Theorems 2 and 3. 
Theorem 2. In the case of increasing market demand, during the ride service time $[0, T]$, for case (i), the optimal dynamic price of the platform $P_{u}^{*}(t)$ is

$$
P_{u}^{*}(t)=\frac{\alpha}{\beta+s r} \cdot e^{-a t}+\frac{\gamma q+s \varepsilon}{\beta+s r} .
$$

The trajectories of the platform's supply rate $S_{u}^{*}(t)$ and demand rate $D_{u}^{*}(t)$ over time are as follows:

$$
\begin{aligned}
& S_{u}^{*}(t)=\frac{s r \alpha}{\beta+s r} \cdot e^{-a t}+\frac{s \cdot(r \gamma q-\beta \varepsilon)}{\beta+s r}, \\
& D_{u}^{*}(t)=\frac{s r \alpha}{\beta+s r} \cdot e^{-a t}+\frac{s \cdot(r \gamma q-\beta \varepsilon)}{\beta+s r} .
\end{aligned}
$$

The proof is in Appendix.

Theorem 2 indicates that the platform's optimal dynamic price, supply rate, and demand rate all change dynamically with time $t$ in the ride service time $[0, T]$. According to Theorem 2, we can obtain some properties of the optimal solution, as in Corollary 3, and the platform's total transaction volume and total profit, as in Corollary 4.

Corollary 3. During the ride service time [0, T], based on Theorem 2, the optimal solution for the ride-hailing platform has the following properties:

(a) $P_{u}^{*}(t)$ is a convex function of time $t$, and $P_{u}^{*}(t)$ increases monotonically

(b) $S_{u}^{*}(t)=D_{u}^{*}(t)$ and $u(t)=0$, so the platform does not have the booking delay cost at time $t$

The proof is in Appendix.

When the ride-hailing platform faces increasing market demand during the ride service time $[0, T]$, we consider that the platform is not constrained by the ceiling price $\left(P_{u}^{*}(t) \leqq \bar{P}\right.$, $\bar{P} \longrightarrow+\infty)$. Therefore, there are enough drivers wanting to join the platform to provide ride service. Thus, the platform can acquire a stable and continuous supply capacity by increasing service price. Corollary 3 indicates that the optimal price $P_{u}^{*}(t)$ increases monotonically with the passage of time in the case of increasing market demand. Thus, the platform can continuously obtain more supply capacity. Moreover, the optimal price $P_{u}^{*}(t)$ can maximally incentivize drivers to join the platform to provide transportation service, which in turn can dynamically affect the supply capacity and reduce the delay in bookings. Therefore, the platform's supply and demand reach a balance, and the delayed bookings reduce to zero.

Corollary 4. During the ride service time [0, T], based on Theorem 2, the platform's total transaction volume $\psi_{u}^{t}$ is

$$
\psi_{u}^{*}(t)=\frac{s r \alpha\left(1-e^{-a T}\right)}{a(\beta+s r)}+\frac{s \cdot(r \gamma q-\beta \varepsilon) T}{\beta+s r} .
$$

The platform's total profit $\pi_{u}^{*}$ is

$$
\pi_{u}^{*}=\int_{0}^{T} S_{u}^{*}(t) \cdot\left((1-r) \cdot P_{u}^{*}(t)-\eta q^{2}\right) \mathrm{d} t .
$$

The proof is in Appendix.
Theorem 3. In case (ii) when the market demand increases during the ride service time [0,T], there exists a critical point $t_{2}=-(1 / a) \ln (\bar{P} \cdot(s r+\beta)-(\gamma q+s \varepsilon) / \alpha)$. If $T \leqq t_{2}$, the optimal dynamic price $P_{u}^{*}(t)$ is calculated as in equation (25). If $0 \leqq t_{2} \leqq T$ and $P_{u}^{\max }=P_{u}^{*}\left(t_{2}\right)=\bar{P}$, thus $P_{u}^{*}(t)$ is as follows:

$$
P_{u}^{*}(t)= \begin{cases}\frac{\alpha}{\beta+s r} \cdot e^{-a t}+\frac{\gamma q+s \varepsilon}{\beta+s r}, & t \in\left[0, t_{2}\right], \\ \bar{P}, & t \in\left(t_{2}, T\right] .\end{cases}
$$

The proof is in Appendix.

Theorem 3 indicates that when the ride-hailing platform faces increasing market demand and the service price is limited by the ceiling price during the ride service time $[0, T]$, the platform's optimal price increases monotonically over time, which is consistent with Corollary 3. Once the optimal price reaches the ceiling price, the platform will achieve maximum supply capacity and the optimal price will not change over time.

According to Theorem 3, we can obtain the trajectories of the platform's supply rate and demand rate over time, as in Corollary 5.

Corollary 5. During the ride service time [0, T], based on Theorem 3, the following can be obtained:

(a) If $T \leqq t_{2}, S_{u}^{*}(t)$ is equal to equation (26), $D_{u}^{*}(t)$ is equal to equation (27), and $S_{u}^{*}(t)=D_{u}^{*}(t)$

(b) If $0 \leqq t_{2} \leqq T, S_{u}^{*}(t)$ is

$$
S_{u}^{*}(t)= \begin{cases}\frac{s r \alpha}{\beta+s r} \cdot e^{-a t}+\frac{s \cdot(r \gamma q-\beta \varepsilon)}{\beta+s r}, & t \in\left[0, t_{2}\right], \\ s r \cdot \bar{P}-s \varepsilon, & t \in\left(t_{2}, T\right] .\end{cases}
$$

$D_{u}^{*}(t)$ is as follows, and $S_{u}^{*}(t) \leqq D_{u^{*}(t)}:$
$D_{u}^{*}(t)= \begin{cases}\frac{s r \alpha}{\beta+s r} \cdot e^{-a t}+\frac{s \cdot(r \gamma q-\beta \varepsilon)}{\beta+s r}, & t \in\left[0, t_{2}\right], \\ \alpha e^{-a t}-\beta \bar{P}+\gamma q, & t \in\left(t_{2}, T\right] .\end{cases}$

The proof is in Appendix.

Corollary 5 shows how the platform's supply rate and demand rate change based on Theorem 3. At first, the platform's supply capacity increases monotonically under the effect of the optimal price, with its supply rate equal to the demand rate to maximize profit. As time passes, due to the constraint of the ceiling price, the platform can acquire limited supply capacity from drivers. However, as the market ride demand increases continuously, the platform will maintain maximum supply capacity to meet ride demand in time and correspondingly reduce the booking delay. The platform must allow for some booking delay cost because delayed bookings 
are unavoidable, which is consistent with the real scenario that, during peak traffic periods, passengers have to wait for rides.

Corollary 6. During the ride service time [0, T], based on Theorem 3 and Corollary 5, the platform's total transaction volume and total profit meet:

(a) If $T \leqq t_{2}$, the total transaction volume $\psi_{u}^{*}$ is equal to equation (28) and the total profit $\pi_{u}^{*}$ is equal to equation (29)

(b) If $0 \leqq t_{2} \leqq T, \psi_{u}^{*}$ is given by

$$
\begin{aligned}
\psi_{u}^{*}(t)= & \frac{s r(\alpha+\gamma q+s \varepsilon-\bar{P}(s r+\beta))}{a(\beta+s r)} \\
& -\frac{1}{a} \cdot \ln \left(\frac{\bar{P} \cdot(s r+\beta)-(\gamma q+s \varepsilon)}{\alpha}\right) \\
& \cdot\left(\frac{s \cdot(r \gamma q-\beta \varepsilon)}{\beta+s r}-(s r \bar{P}-s \varepsilon) \bar{P}\right)+(s r \bar{P}-s \varepsilon) T .
\end{aligned}
$$

The platform's total profit $\pi_{u}^{*}$ is given by

$$
\begin{aligned}
\pi_{u}^{*}= & \int_{0}^{t_{2}} S_{u}^{*}(t) \cdot\left((1-r) \cdot P_{u}^{*}(t)-\eta q^{2}\right) \mathrm{d} t \\
& +\int_{t_{2}}^{T} \int_{t_{2}}^{T}\left[S_{u}^{*}(t) \cdot\left((1-r) \cdot \bar{P}-\eta q^{2}\right)\right. \\
& \left.-h \cdot \int_{t_{2}}^{t}\left(D_{u}^{*}(\tau)-S_{u}^{*}(\tau)\right) \mathrm{d} \tau\right] \mathrm{d} t .
\end{aligned}
$$

The proof is in Appendix.

4.3. Optimal Pricing Strategy with Stable Market Demand $(a=0)$. In this section, we consider the scenario in which the market demand is stable, that is, a scenario between a high demand state and a low demand state with no fluctuation during the period. In practice, sometimes the number of riders who need to take a trip is almost equal to the number of the drivers in a certain area. Therefore, in this case, the supply and demand balance of the ride-hailing platform at time 0 remains the same, and there is no opportunity cost or booking delay cost during the ride service time $[0, T]$. This situation can be modeled as follows.

The objective function is given by

$$
\begin{aligned}
\pi_{b}\left(P_{b}, t\right) & =\max _{P_{b}(t)} \int_{0}^{T} \int_{0}^{T}\left[D_{b}\left(P_{b}, t\right) \cdot P_{b}(t)-D_{b}\left(P_{b}, t\right) \cdot C_{b}(t)\right] \mathrm{d} t \\
& =\max _{P_{b}(t)} \int_{0}^{T}\left[D_{b}(P, t) \cdot\left(P_{b}(t)-W_{b}(t)-\eta q^{2}\right)\right] \mathrm{d} t .
\end{aligned}
$$

The constraints are

$$
\begin{aligned}
v(t) & =u(t)=0 \\
a & =0 .
\end{aligned}
$$

Theorem 4. During the ride service time [0, T], the market demand is stable. The platform's optimal dynamic price $P_{b}^{*}(t)$ is

$$
P_{b}^{*}(t)=\frac{\alpha}{s r+\beta}+\frac{\gamma q+s \varepsilon}{s r+\beta}
$$

The trajectories of the platform's supply rate $S_{b}^{*}(t)$ and demand rate $D_{b}^{*}(t)$ over time are

$$
\begin{aligned}
& S_{b}^{*}(t)=\frac{s r \alpha}{s r+\beta}+\frac{s \cdot(r \gamma q-\beta \varepsilon)}{s r+\beta}, \\
& D_{b}^{*}(t)=\frac{s r \alpha}{s r+\beta}+\frac{s \cdot(r \gamma q-\beta \varepsilon)}{s r+\beta} .
\end{aligned}
$$

The proof is in Appendix.

According to Theorem 4, the optimal price does not change dynamically with time $t$ when the market demand is stable during the ride service time $[0, T]$. The optimal price is also a constant, and the platform retains a certain number of drivers to provide transport and balance the market demand to ensure smooth operation.

Based on the optimal solution of Theorem 4, the platform's total transaction volume and total profit can be obtained, as in Corollary 7.

Corollary 7. During the ride service time [0,T], when the market demand is stable, the platform's total transaction volume $\psi_{b}^{*}$ is

$$
\psi_{b}^{*}(t)=s T \cdot \frac{(r \alpha+r \gamma q-\beta \varepsilon)}{(s r+\beta)} .
$$

The platform's total profit $\pi_{b}^{*}$ is

$$
\pi_{b}^{*}=\int_{0}^{T} D_{b}^{*}(t) \cdot\left((1-r) \cdot P_{b}^{*}(t)-\eta q^{2}\right) \mathrm{d} t .
$$

The proof is in Appendix.

Next, according to the modeling and the analysis above, we summarize in Table 2, the properties of the optimal dynamic prices in the three scenarios, where $\uparrow$ indicates the variable is increasing over time, $\longrightarrow$ indicates the variable is stable or unchanged over time, and $\downarrow$ indicates the variable is decreasing over time.

4.4. Impact of Key Parameters on the Optimal Pricing Strategies. Some key parameters - such as the coefficient of demand fluctuation $a$, service quality $q$, and fixed commission rate $r$-have an important impact on the platform's pricing decisions. In this section, we analyze these parameters' impact on the platform's optimal pricing strategies based on the optimal solutions in Sections 4.1, 4.2, and 4.3. Thus, we try to answer the following questions: how does the platform dynamically adjust its pricing decisions based on the changes in the degree of market demand fluctuation? How do the platform's service quality and fixed commission contract affect pricing decisions under different market demand situations? We divide our analysis into three sections. In Section 4.4.1, we study the impact of $a$. In Section 
TABLe 2: The optimal dynamic price changes with $t$.

\begin{tabular}{|c|c|c|c|}
\hline $\begin{array}{l}\text { Market demand } \\
\text { situations }\end{array}$ & Cases & T's conditions & $P^{*}(t)$ \\
\hline \multirow{4}{*}{$a>0$} & \multirow[t]{2}{*}{$c=0$} & $\begin{array}{l}T \leqq T_{\text {th }} \\
T>T_{\text {t }}\end{array}$ & $\downarrow$ \\
\hline & & $\begin{array}{c}T>I_{\text {th }} \\
T \leqq t_{1}\end{array}$ & $\downarrow$ \\
\hline & $c \neq 0$ & $T>t_{1}$ & $\downarrow \uparrow$ \\
\hline & $\bar{P} \longrightarrow+\infty$ & & $\uparrow$ \\
\hline$a<0$ & $\bar{P}$ is a constant & $\begin{array}{l}T \leqq t_{2} \\
T>t_{2}\end{array}$ & $\uparrow \stackrel{\uparrow}{\uparrow}$ \\
\hline$a=0$ & & & \\
\hline
\end{tabular}

4.2.2, we analyze the impact of $q$. In Section 4.4.3, we discuss the impact of $r$. In Section 4.4, for the convenience of analysis and discussion, we consider the general cases in which we assume $c>0, \bar{P}$ is a constant, and $t_{1}, t_{2}<\mathrm{T}$. Furthermore, as $P_{u}^{*}(t)=\bar{P}$ when $t \in\left[t_{2}, T\right]$, we only consider $t \in\left[0, t_{2}\right]$ in the case of increasing market demand.

4.4.1. Coefficient of Demand Fluctuation. In this section, we analyze how parameter $a$ affects the platform's pricing decision under both scenarios of decreasing and increasing fluctuating market demand. Usually, the degree of market demand fluctuation varies in different regions; for example, there is more ride-hailing demand in certain central business districts of Shanghai. Of course, even in the same region, the degree of market demand fluctuation at different times might vary, such as during rainy weather. Therefore, to maximize the profit, the platform adjusts its optimal pricing strategies dynamically according to the different degrees of market demand fluctuation. For example, Uber's current pricing engine generates tens of millions of price decisions at the level of geographic units every minute across the world.

Proposition 1. During the ride service time [0,T], the impact of $a$ on the optimal pricing strategies as follows:

(a) In decreasing market demand, $P_{v}^{*}(t)$ decreases when $a$ increases. There is a critical point $a^{*}=$ $(2 c e(s r+\beta) / \alpha(1-r))$. If $a \leqq a^{*}, t_{1}$ increases when $a$ increases. If $a>a^{*}, t_{1}$ decreases when a increases.

(b) In increasing market demand, during the ride service time $\left[0, t_{2}\right]$, when a decreases, $P_{u}^{*}(t)$ increases and $t_{2}$ decreases.

According to Proposition 1, the influence of $a$ on the platform's pricing decisions can be summed up as follows: the faster the decrease in market demand, the lower the optimal price. If the coefficient of demand fluctuation $a$ is smaller than the critical value $a^{*}$ and the platform's price reaches $P_{v}^{\min }$, the time point $t_{1}$ moves backward when $a$ increases. However, if the coefficient of demand fluctuation $a$ is higher than the critical value $a^{*}$ and the platform reaches $P_{v}^{\min }$, the time point $t_{1}$ moves forward when $a$ increases. The faster the market demand increases, the higher the optimal price when the platform's price is not at its ceiling price, and the time point $t_{2}$ moves forward when the platform's price reaches $P_{u}^{\max }$.
4.4.2. Service Quality. The platform's service quality includes passenger satisfaction with the ride service. For example, China's DiDi platform usually adjusts the matching distance between passengers and surrounding drivers based on the demand situation in a certain area, which influences the time it takes for the driver to pick up the passenger and thus affects customer satisfaction. Passengers are concerned not only with the price but also with the quality of the service. Passenger satisfaction is related to the platform's service quality. However, from the platform's perspective, service quality is closely related to service cost, which affects pricing decisions. Therefore, it is important to discuss the service quality and determine how the service quality affects the optimal pricing strategies. This section analyzes how service quality parameter $q$ affects the platform's pricing decisions under three market situations: decreasing demand, increasing demand, and stable demand.

Proposition 2. During the ride service time [0, T], the impact of service quality $q$ on the optimal pricing strategies is as follows:

(a) In the three market demand situations (i.e., $a>0$, $a<0, a=0)$ and $t \in\left[0, t_{2}\right]$ when market demand increases, the optimal price $P^{*}(t)$ increases when $q$ increases. For each unit of increase in $q, P^{*}(t)$ increases by $\gamma /(\beta+s r)$

(b) In decreasing market demand, $t_{1}$ remains unchanged when $q$ decreases

(c) In increasing market demand, $t_{2}$ increases when $q$ decreases

Proposition 2 indicates that regardless of any market demand situation, the platform's optimal prices rise as service quality increases. In reality, when service quality improves, it is because the platform usually has invested more human and technical resources, which inevitably increase operation costs. As a result, service prices rise accordingly to maximize the platform's profit. In addition, when q decreases, the time point $t_{1}$ is unchanged when the platform's price reaches $P_{v}^{\min }$, but the time point $t_{2}$ moves backward when the platform's price reaches $P_{u}^{\max }$. In other words, the service period $t_{2}$ to $T$ represents the peak congestion period. During this time, the platform might correspondingly reduce the peak congestion period if $q$ is appropriately reduced, to decrease part of the ride demand and thus reduce the time spent in traffic jams.

4.4.3. Fixed Commission Rate. The fixed commission rate is the percentage of the fare that the platform pays to the drivers after they complete each ride. Setting a fixed commission contract not only directly affects the platform's revenue from each transaction but also affects the drivers' income. In reality, the platform's fixed commission rate might change; for example, Didi Chuxing's fixed commission rate varies from approximately 0.7 to 0.8 . Thus, to maximize the platform's profits, it is necessary to adjust the optimal pricing strategies by setting different commission rates. In this section, we analyze how the fixed commission rate parameter $r$ affects the platform's pricing decisions 
under three market demand situations. For the convenience of analysis, we assume $r_{h}$ means the platform adopts a higher fixed commission rate, and $r_{l}$ means the platform adopts a lower fixed commission rate, and $r_{h}>r_{l}$.

Proposition 3. During the ride service time [0,T], $r_{h}$ is the higher fixed commission rate, $r_{l}$ is the lower fixed commission rate, and $r_{h}>r_{l}$. The platform adjusts its optimal pricing strategies with different $r$ :

(a) In decreasing market demand, we have a critical point $t_{3}=\left(\beta s(\alpha+\gamma q+s \varepsilon) \cdot(1-r)^{2} / c(s+\beta) \cdot(s r+\beta)^{2}\right)$, when $t<t_{3}, \quad P_{v}^{*}\left(r_{h}, t\right)<P_{v}^{*}\left(r_{l}, t\right)$. When $t=t_{3}$, $P_{v}^{*}\left(r_{h}, t_{3}\right)=P_{v}^{*}\left(r_{l}, t_{3}\right)$. When $t>t_{3}$, $P_{v}^{*}\left(r_{h}, t_{3}\right)>P_{v}^{*}\left(r_{l}, t_{3}\right)$. Additionally, we have $t_{1}\left(r_{h}\right)<t_{1}\left(r_{l}\right)$.

(b) In increasing market demand during the ride service time $\left[0, t_{2}\right], P_{u}^{*}\left(r_{h}, t\right)<P_{u}^{*}\left(r_{l}, t\right), t_{2}\left(r_{h}\right)>t_{2}\left(r_{l}\right)$.

(c) In stable market demand, $P_{b}^{*}\left(r_{h}, t\right)<P_{b}^{*}\left(r_{l}, t\right)$.

According to Proposition 3, the effects of $r$ on the platform's pricing decisions can be summarized as follows: the optimal price is higher when the platform reduces the fixed commission rate $r$. This result is intuitive because the decrease in $r$ leads to less supply capacity, whereas higher prices can change the situation. However, for decreasing market demand, once demand drops to a certain level, the platform has to increase $r$, and thus the optimal price is higher. The platform faces deteriorating market demand, but once $r$ increases, it leads to lower revenue for each transaction; thus, the platform raises the optimal price to ensure revenue. In addition, when $r$ increases, the time point $t_{1}$ moves forward when the platform's price reaches $P_{v}^{\min }$, but the time point $t_{2}$ moves backward when the platform reaches $P_{u}^{\max }$. In other words, as the service period $t_{2}$ to $T$ represents the peak congestion period, the platform can correspondingly reduce the peak congestion period by appropriately improving $r$.

Tables 3 and 4 summarize the impact of the key parameters on pricing decisions based on the analysis above and Propositions $1-3$.

\section{Extended Model}

From a long cycle perspective, the ride demand may first increase and then decrease, or first decrease and then increase, so the benchmark model may not be intuitive. In this section, we construct a general model to extend our benchmark model in Section 4, which can provide reference for the ride-hailing platform enterprises when the situation in which there is increasing or decreasing fluctuation of market demand (e.g., first increasing and then decreasing). In addition, we find the characteristics of the optimal dynamic price trajectory in the general model are consistent with those of the benchmark model, that is, the other forms of requirement functions will not change the correctness of the conclusion.

To facilitate the construction of the general model, we adjusted the assumptions of the benchmark model as follows: 1. assumption 7 is removed, which means that the initial demand of the market (i.e., $\alpha(t)$ ) in the demand
TABLE 3: The impact of the key parameters on optimal prices.

\begin{tabular}{|c|c|c|c|c|c|c|}
\hline \multirow{2}{*}{ Optimal price } & \multirow{2}{*}{$a \uparrow(a>0)$} & \multirow{2}{*}{$a \downarrow(a<0)$} & \multirow{2}{*}{$q \uparrow$} & \multicolumn{3}{|c|}{$r \downarrow$} \\
\hline & & & & $t<t_{3}$ & $t=t_{3}$ & $t>t_{3}$ \\
\hline$P_{v}^{*}(t)$ & $\downarrow$ & - & $\uparrow$ & $\uparrow$ & $\longrightarrow$ & $\downarrow$ \\
\hline$P_{u}^{*}(t)$ & - & $\uparrow$ & $\uparrow$ & $\uparrow$ & $\uparrow$ & $\uparrow$ \\
\hline$P_{b}^{*}(t)$ & - & - & $\uparrow$ & $\uparrow$ & $\uparrow$ & $\uparrow$ \\
\hline
\end{tabular}

TABLE 4: The impact of the key parameters on critical points.

\begin{tabular}{lccccc}
\hline Critical point & \multicolumn{2}{c}{$a \uparrow(a>0)$} & & & \\
& $a \leqq a^{*}$ & $a>a^{*}$ & $a \downarrow(a<0)$ & $q \downarrow$ & $r \uparrow$ \\
\hline$t_{1}$ & $\uparrow$ & $\downarrow$ & - & $\longrightarrow$ & $\downarrow$ \\
$t_{2}$ & - & - & $\downarrow$ & $\uparrow$ & $\uparrow$ \\
\hline
\end{tabular}

function can be non-monotonic; 2 . from a long cycle perspective, changes in the supply and demand state of the platform will be difficult to control and model, so we assumed that all ride requests can be satisfied eventually (even if the ride bookings are delayed during peak demand) at time $t$, that is, we consider the demand rate to be fully satisfied at time $t$, where there is no booking delay or opportunity cost (in this section, the authors focus on studying the effect of the non-monotonic demand fluctuation on optimal pricing problem of the ride-hailing platform, without considering the supply-side characteristics of the platforms. The platform's opportunity cost and booking delay cost can be viewed as endogenously determined by setting the price, which does not affect the analysis in this section).

The ride-hailing platform's objective is to find the optimal dynamic price trajectory when the market demand fluctuation is uncertain to maximize the total profit over the ride service time of $[0, \mathrm{~T}]$. The objective function is given as follows:

$$
\begin{aligned}
\pi(P, t) & =\max _{P(t)} \int_{0}^{T}[D(P, t) \cdot P(t)-D(P, t) \cdot C(t)] \mathrm{d} t, \\
& =\max _{P(t)} \int_{0}^{T}\left[(\alpha(t)-\beta P(t)+\gamma q) \cdot\left(P(t)-W(t)-\eta q^{2}\right)\right] \mathrm{d} t,
\end{aligned}
$$

where $\alpha(t)$ changes over time is uncertain, it can be nonmonotonic. With the support of big data and artificial intelligence technology (e.g., DiDi Brain, that is, an artificial intelligence brain based on traffic data is established by DiDi in the cloud), the platform can predict the fluctuation of the ride demand market in a certain service region based on historical data.

Observe that, in the general model, the solution of the optimal dynamic price trajectory of the ride-hailing platform can be regarded as a functional extremal problem. According to equation (42), the optimal control variational method is used to solve the extremal value, denoted as follows:

$$
F=(\alpha(t)-\beta P(t)+\gamma q) \cdot\left(P(t)-W(t)-\eta q^{2}\right) .
$$

Lemma 3. During the ride service time $[0, T]$, with uncertain market demand fluctuation, the platform's profit $\pi(P, t)$ is 
strictly concave in price $P(t)$, and the optimal dynamic price solution $P^{*}(t)$ can maximize the platform's profit $\pi(P, t)$.

Proof. From equation (43), the second partial derivative of $F$ with respect to $P(t)$ can be calculated as follows:

$$
\frac{\mathrm{d}^{2} F}{\mathrm{~d} P^{2}}=-2 \beta(1-r)
$$

Since $\beta>0,0<r<1$, then by equation (44), we have $\left(\mathrm{d}^{2} F / \mathrm{d} P^{2}\right)<0$, which indicates that $\pi(P, t)$ is a concave function of $P(t)$. Obviously, we can get Lemma 3 .

Thus, Lemma 3 is proved.

In order to maximize the profit with uncertain market demand fluctuation, according to the necessary condition for an extremum of the functional, we have the Euler equation, that is, $(\partial F / \partial P(t))-(\mathrm{d} / \mathrm{d} t)(\partial F / \partial P \prime(t))=0$. Since there is no $P I(t)$ term in equation (43), the Euler equation can be simplified to $(\mathrm{d} F / \mathrm{d} P(t))=0$. By solving this firstorder condition, Theorem 5 can be obtained.

Theorem 5. During the ride service time [0,T], with uncertain market demand fluctuation, the ride-hailing platform's optimal dynamic price trajectory $P^{*}(t)$ is

$$
P^{*}(t)=\frac{\alpha(t)+\gamma q}{2 \beta}+\frac{\eta q^{2}}{2(1-r)},
$$

where if we consider the minimum wage constraint (i.e., $W(t)>\varepsilon$ ) and the price ceiling $\bar{P}$, the optimal dynamic price trajectory satisfies $\varepsilon / r<P^{*}(t) \leqq \bar{P}$.

Theorem 5 indicates that the optimal dynamic price trajectory of ride-hailing platform changes dynamically with time $t$. Intuitively, the characteristics of the optimal dynamic price trajectory $P^{*}(t)$ changing with time are positively correlated with the fluctuation of potential market demand $\alpha(t)$ with time. Therefore, the optimal dynamic price will change dynamically with the uncertain fluctuation of market demand during the ride service time $[0, T]$. For example, when the market demand is decreasing in a short period of time, the optimal dynamic price trajectory $P^{*}(t)$ decreases monotonically over time, so as to alleviate the weakening market demand and to stimulate market demand as much as possible to maximize the utilization of supply capacity, which is consistent with the benchmark model of Section 4.1. In the same way, when the market demand is increasing in a short period of time, the optimal dynamic price trajectory $P^{*}(t)$ increases monotonically over time, which is consistent with the benchmark model of Section 4.2. As we can see, the other forms of requirement functions (e.g., the market demand rate fluctuations are not driven by the exponential process) will not change the correctness of the conclusion.

\section{Numerical Analysis}

In this section, we conduct numerical studies to verify the conclusions obtained in the previous sections. We also draw some new conclusions by analyzing the impact of the key parameters on the platform's total transaction volume and total profit under different market demand situations. For simplicity, we assume the driver's minimum participation cost per time unit $\varepsilon=0$. This assumption does not affect the results. As in [8] and [16], for the numerical analysis, we first provide the parameters (Table 5) used in the different scenarios.

6.1. Optimal Solutions. In Sections 4.1, 4.2, and 4.3, we present the optimal solutions for the pricing decisions of three market demand situations: decreasing, increasing, and stable. The three situations are examined in this section. In addition to the fixed parameters in Table 5 , the other parameters are $q=30, r=0.7$, and $a=\{0.03,-0.03,0\} ; a=0.03$ indicates that the platform faces decreasing market demand, $a=-0.03$ indicates that the platform faces increasing market demand, and $a=0$ indicates that the platform faces stable market demand.

First, we analyze the scenario of decreasing market demand during the ride service time $[0, T]$. According to Theorem 1, Figure 5(a) shows the optimal price trajectory when the platform's demand decreases first and then increases. This result is consistent with the general case of $c \neq 0$ in Corollary 1.

Here, the corresponding optimal dynamic price is

$$
P_{v}^{*}(t)=50 e^{-0.03 t}+0.8 t-6.25, \quad t \in[0,30] \text {. }
$$

Figure 5(b) shows the trajectories of demand rate and supply rate under the influence of optimal prices. In Figure 5(b), area $N$ indicates that the platform reduces the supply capacity to appropriately avoid too many drivers accepting bookings on the platform; area $M$ indicates that the optimal price alleviates the attenuation of market demand to some extent; and area $Q$ indicates that the platform does not achieve a balance of supply and demand under profit maximization, or that the platform is not aiming to eliminate excess supply capacity. From Figure 5(c), we see that the platform's reduced profit rate with time cannot offset the impact of weakening market demand on the platform's profit, even with the price increase in the later service period.

Next, we look at the second scenario-increasing market demand during the ride service time $[0, T]$. This research only simulates the case of the platform being constrained by the ceiling price $\left(P_{u}(t) \leqq \bar{P}, \bar{P}\right.$ is a constant). Figure 6(a) shows that the platform's optimal price increases first and then remains unchanged, which is consistent with the analysis of Theorem 3. The corresponding optimal dynamic price is

$$
P_{u}^{*}(t)= \begin{cases}41.67 e^{0.03 t}+2.086, & t \in[0,20.86], \\ 80, & t \in(20.86,30] .\end{cases}
$$

Figure 6(b) shows the trajectories of demand rate and supply rate under the influence of optimal prices. In Figure 6(b), area $X$ indicates that, although the increase in price decreases the demand, it can satisfy the demand of people who are more anxious for a ride service; area $Y$ indicates that the price increase greatly increases the platform's supply capacity, which can satisfy the ride demands of more people in that period; and area $Z$ indicates that the 
TABLE 5: Tested parameter values.

\begin{tabular}{lccccccccc}
\hline Parameter & $T$ & $\alpha$ & $\beta$ & $\gamma$ & $s$ & $\eta$ & $C$ & $h$ & $\bar{P}$ \\
\hline Value & 30 & 400 & 4 & 1 & 8 & 0.001 & 0.1 & 0.1 & 80 \\
\hline
\end{tabular}

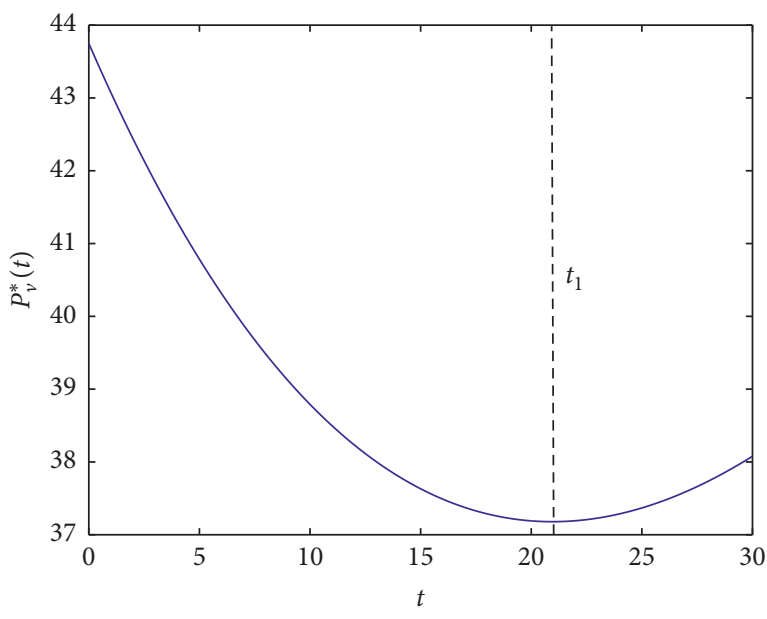

(a)

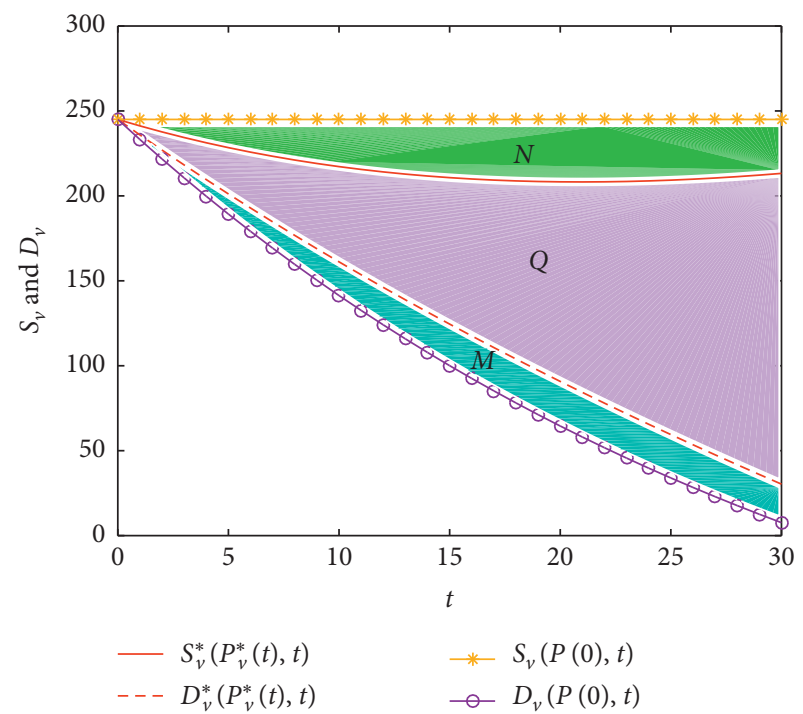

(b)

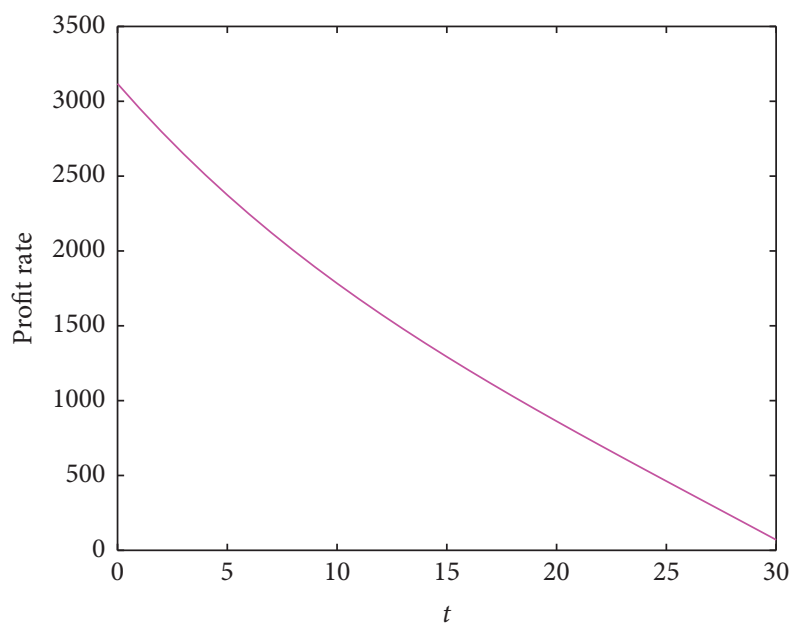

(c)

Figure 5: (a) Optimal price trajectory, (b) the trajectories of demand and supply, and (c) the trajectory of the profit rate in decreasing market demand $(a>0)$.

platform faces the peak congestion period because of the ceiling price. From Figure 6(c), we see that the platform's profit rate first increases and then stabilizes.

Finally, we examine the scenario of stable market demand during the ride service time $[0, T]$. As shown in Theorem 4 and Figures 7(a) and 7(b), the trajectories of the optimal price, demand rate, and supply rate are unchanged over time. Here, the corresponding optimal dynamic price is

$$
P_{b}^{*}(t)=43.75, \quad t \in[0,30] .
$$

In addition, Figure 7(c) shows that the platform's profit rate remains unchanged over time.

\subsection{Key Parameters}

6.2.1. Coefficient of Demand Fluctuation (a). In addition to the fixed parameters in Table 5, other parameters are $q=30$, $r=0.7$, and $a=\{0.025,0.03,0.04,-0.025,-0.03,-0.04\} . a=$ $\{0.025,0.03,0.04\}$ indicates the different degrees of decreasing market demand and $a=\{-0.025,-0.03,-0.04\}$ indicates the different degrees of increasing market demand. In decreasing market demand, a greater value $a$ represents a faster decrease for market demand. In increasing market demand, a smaller value $a$ represents a faster increase for market demand. Figure 8(a) shows the curves of optimal dynamic prices with different $a$ values when market demand 


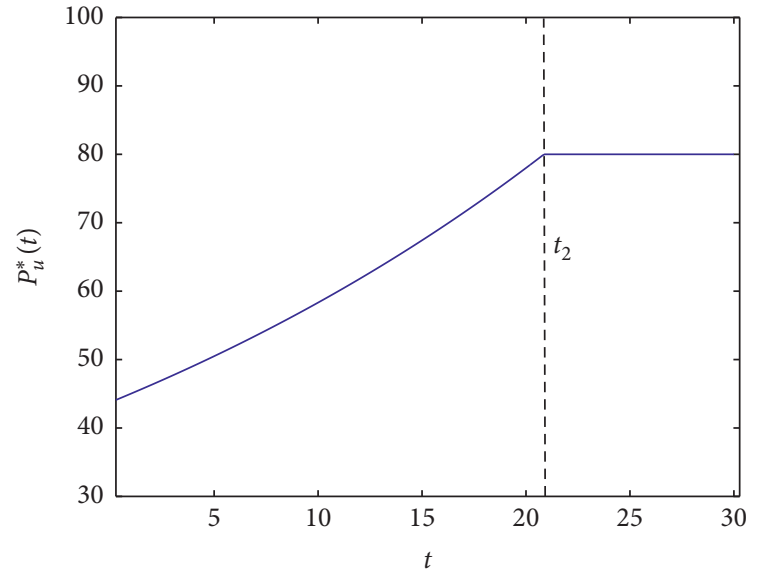

(a)

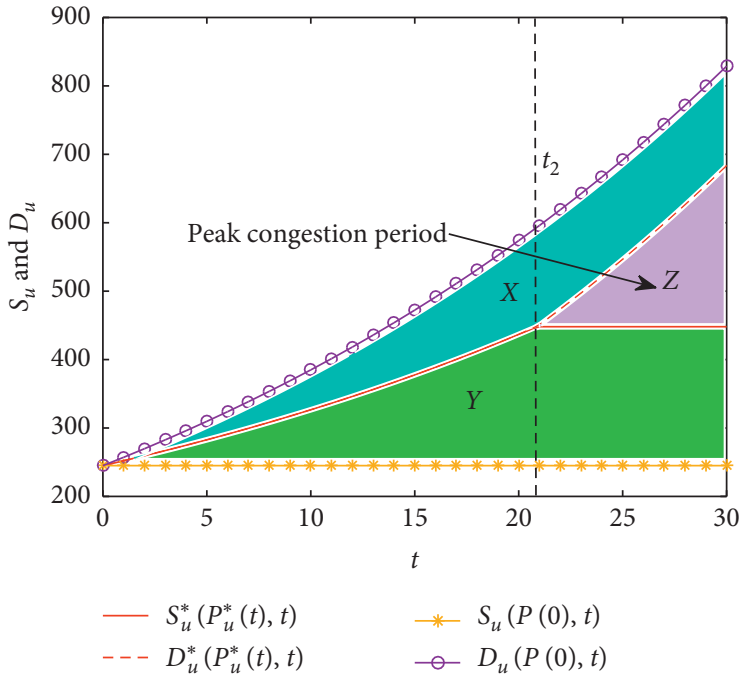

(b)

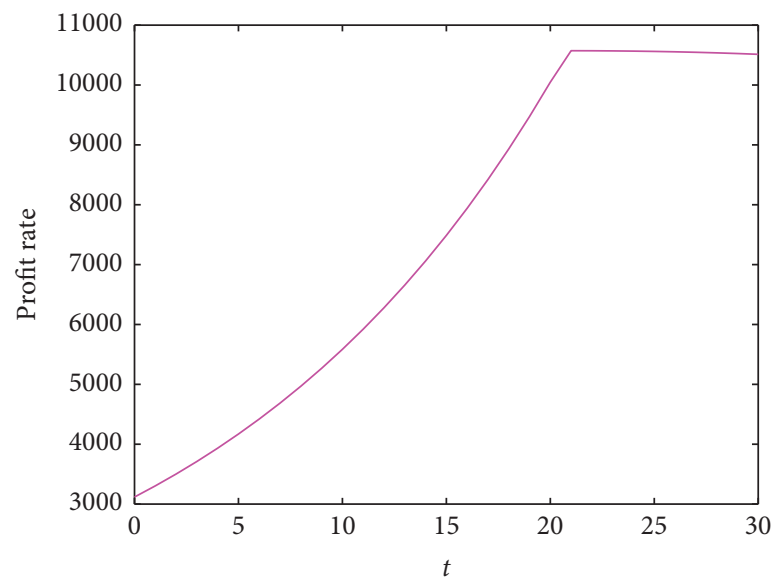

(c)

Figure 6: (a) Optimal price trajectory, (b) the trajectories of demand and supply, and (c) the trajectory of the profit rate in increasing market demand $(a<0)$.

is decreasing. It is shown from Figure 8(a) that, for a greater value $a$, the platform's price is relatively lower and the time point $t_{1}$ will move backward since $a<a^{*}=0.043$ in this scenario, which is consistent with Proposition 1 (a). Figure 8(b) shows the curves of optimal dynamic prices with different $a$ values when market demand increases. In Proposition 1 (b), it is shown from Figure 8(b) that, for a smaller value $a$, the platform's price is relatively higher and the time point $t_{2}$ moves forward, which means the platform will face a longer peak congestion period.

From Table 6, we observe the following:

(i) With decreasing market demand in the ride service time $[0, T]$, the platform's total transaction volume and total profit both decrease when $a$ increases. In other words, a faster decrease in market demand results in lower transaction volume and profit.

(ii) With increasing market demand in the ride service time $[0, T]$, the platform's total transaction volume and total profit both increase when $a$ decreases. In other words, a faster increase in market demand results in relatively higher transaction volume and profit.

6.2.2. Service Quality (q). In addition to the fixed parameters in Table 5, other parameters are $q=\{10,30,50\}$, $r=0.7$, and $a=\{0.03,-0.03,0\} . q=\{10,30,50\}$ indicates the platform may adopt different service qualities. Figure 9 shows that the optimal prices increase with an increase in $q$ regardless of market demand situations when the platform is not constrained by the ceiling price, which is consistent with Proposition 2 (a). In addition, by observing Figure 9(a), the time point $t_{1}$ is unchanged with a different $q$ value, which is consistent with Proposition 3 (b). As depicted in Proposition 2 (c), Figure 9(b) shows that the time point $t_{2}$ will move backward when $q$ decreases, and $t_{2}$ is pushed backward, meaning the peak congestion period is reduced. 

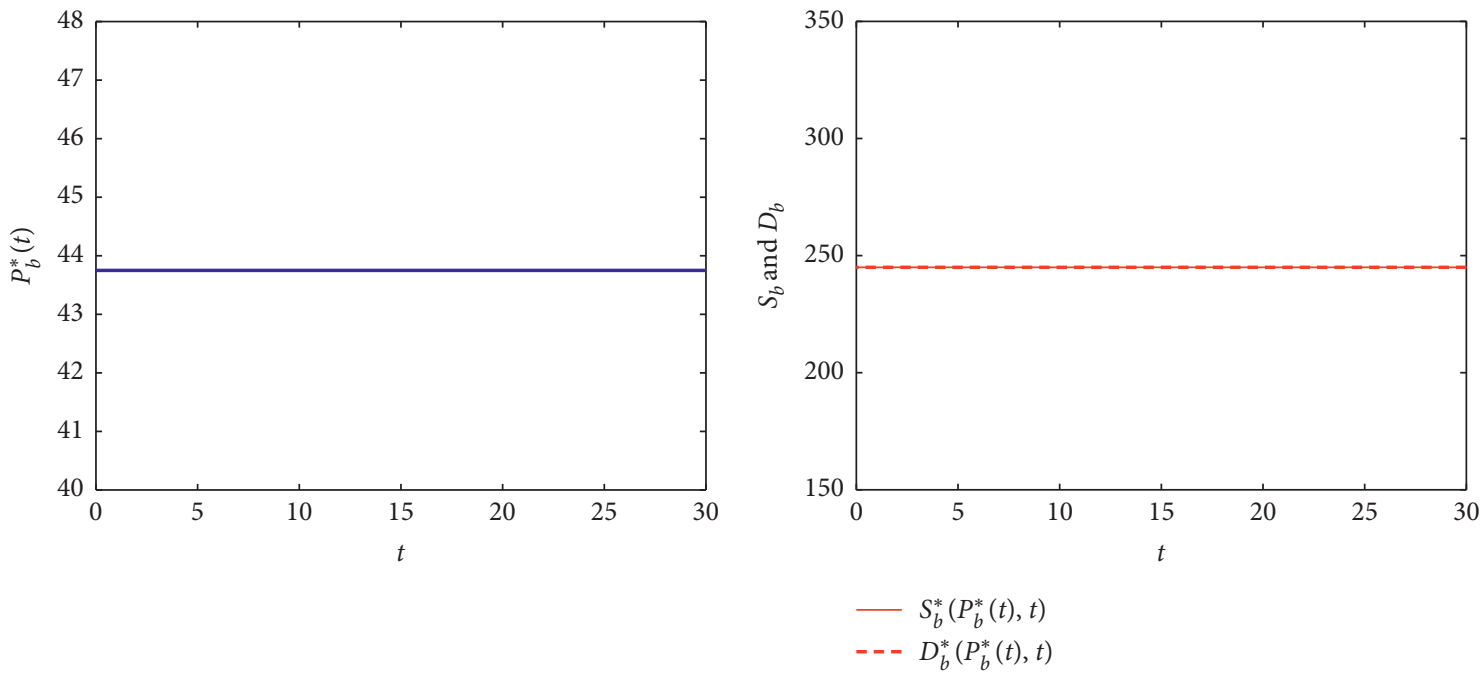

(a)

(b)

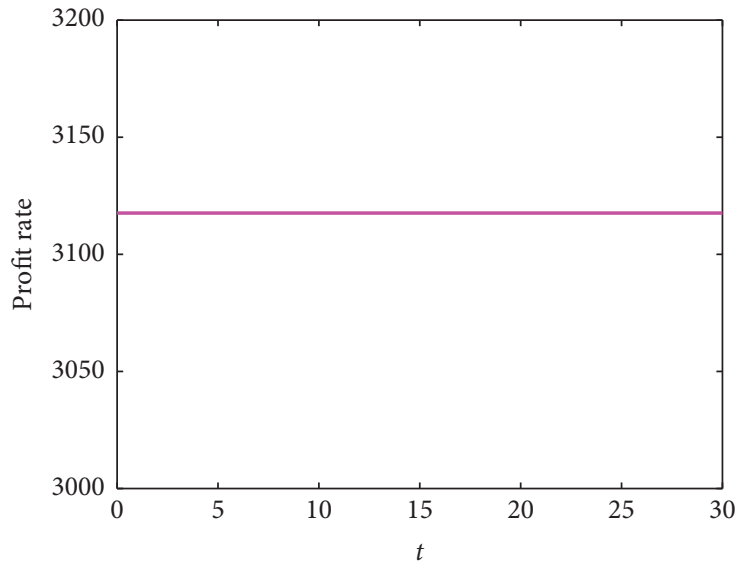

(c)

Figure 7: (a) Optimal price trajectory, (b) the trajectories of demand and supply, and (c) the trajectory of the profit rate in decreasing market demand $(a=0)$.

From Table 7, we observe the following:

(i) Regardless of market demand, the platform's total transaction volume increases when $q$ increases.

(ii) For the same service quality, the total transaction volume under increasing market demand is higher than under stable market demand, and the volume under stable market demand is higher than under decreasing market demand. That is, $\psi_{u}^{*}(q)>\psi_{b}^{*}(q)>\psi_{v}^{*}(q)$.

(iii) Regardless of market demand, the platform's total profit increases first and then decreases when $q$ increases. The total profit is the largest when $q=40$ under decreasing market demand, that is, $\pi_{v}^{*}\left(q_{v}^{*}=40\right) \geqq \pi_{v}^{*}(q)$. The total profit is the largest when $q=30$ under stable market demand, that is, $\pi_{b}^{*}\left(q_{b}^{*}=30\right) \geqq \pi_{b}^{*}(q)$. The total profit is the largest when $q=20$ under increasing market demand, that is, $\pi_{u}^{*}\left(q_{u}^{*}=20\right) \geqq \pi_{u}^{*}(q)$. Furthermore, we find the service quality of the total profit maximized under decreasing market demand is higher than under stable market demand, and the service quality under stable market demand is higher than under increasing market demand. That is, $q_{v}^{*}=40 \geq q_{b}^{*}=30>q_{u}^{*}=20$.

(iv) For the same service quality, the total profit under increasing market demand is higher than under stable market demand, and the profit under stable market demand is higher than under decreasing market demand. That is, $\pi_{u}^{*}(q)>\pi_{b}^{*}(q)>\pi_{v}^{*}(q)$.

6.2.3. Fixed Commission Rate ( $r$ ). In addition to the fixed parameters in Table 5, other parameters are $q=30, r=\{0.5$, $0.7\}$, and $a=\{0.03,-0.03,0\}$. Hu and Zhou [4] obtained the optimal fixed commission rate of 0.6063 . We set $r=\{0.5,0.7\}$ to compare the scenarios when the platform adopts different commission rates. As shown in Proposition 3 (a), for 


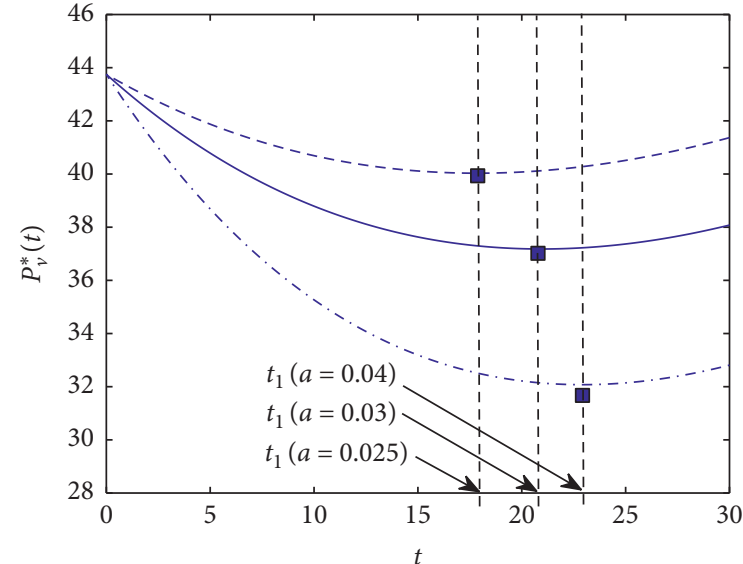

$$
\begin{aligned}
-- & a=0.025 \\
- & a=0.03 \\
-- & a=0.04
\end{aligned}
$$

(a)

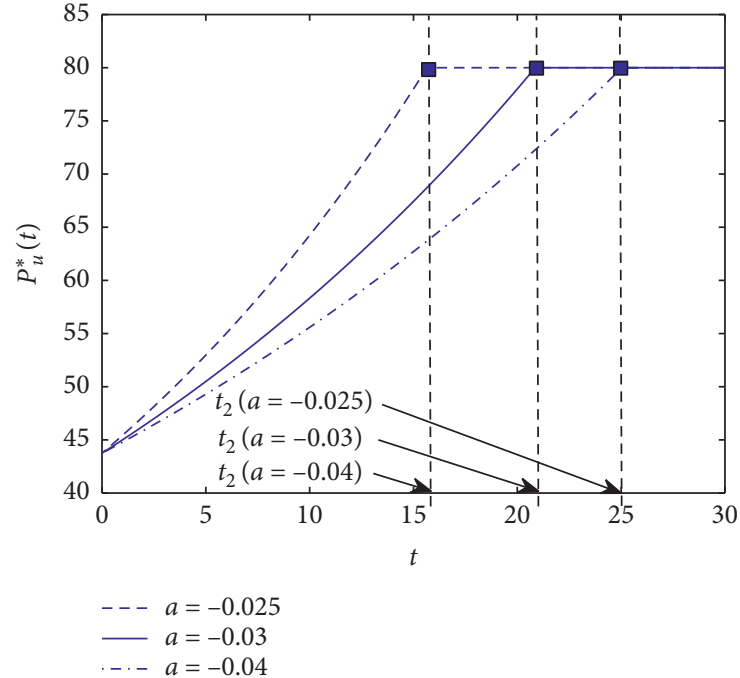

(b)

\begin{tabular}{|c|c|c|c|c|c|}
\hline$a$ & $\psi_{v}^{*}\left(\times 10^{3}\right)$ & $\pi_{v}^{*}\left(\times 10^{4}\right)$ & $a$ & $\psi_{u}^{*}\left(\times 10^{3}\right)$ & $\pi_{u}^{*}\left(\times 10^{4}\right)$ \\
\hline 0.015 & 4.74 & 5.98 & -0.015 & 8.77 & 13.05 \\
\hline 0.02 & 4.42 & 5.27 & -0.02 & 9.93 & 17.71 \\
\hline 0.025 & 4.13 & 4.67 & -0.025 & 10.64 & 20.51 \\
\hline 0.03 & 3.87 & 4.17 & -0.03 & 11.10 & 22.36 \\
\hline 0.035 & 3.62 & 3.74 & -0.035 & 11.44 & 23.66 \\
\hline 0.04 & 3.40 & 3.37 & -0.04 & 11.69 & 24.61 \\
\hline
\end{tabular}

Figure 8: Optimal dynamic prices with different $a$ values under different market situations. (a) $a>0$. (b) $a<0$.

TABLE 6: The impact of $a$ values on the platform's total transaction volume and total profit under decreasing and increasing market demand.

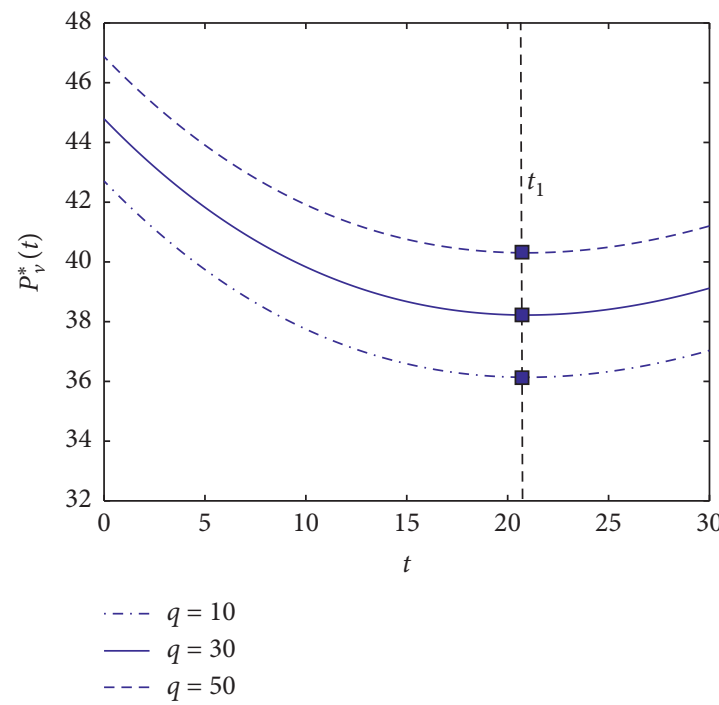

(a)

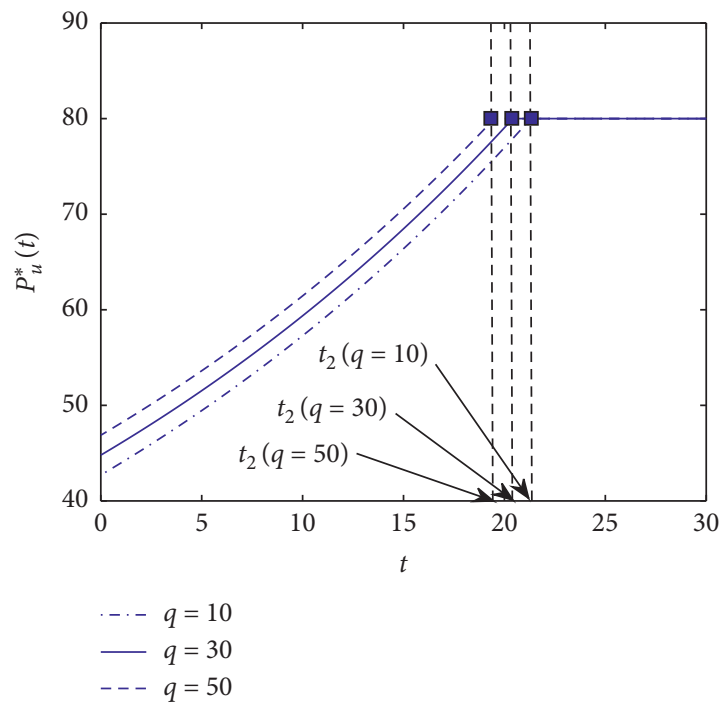

(b)

Figure 9: Continued. 


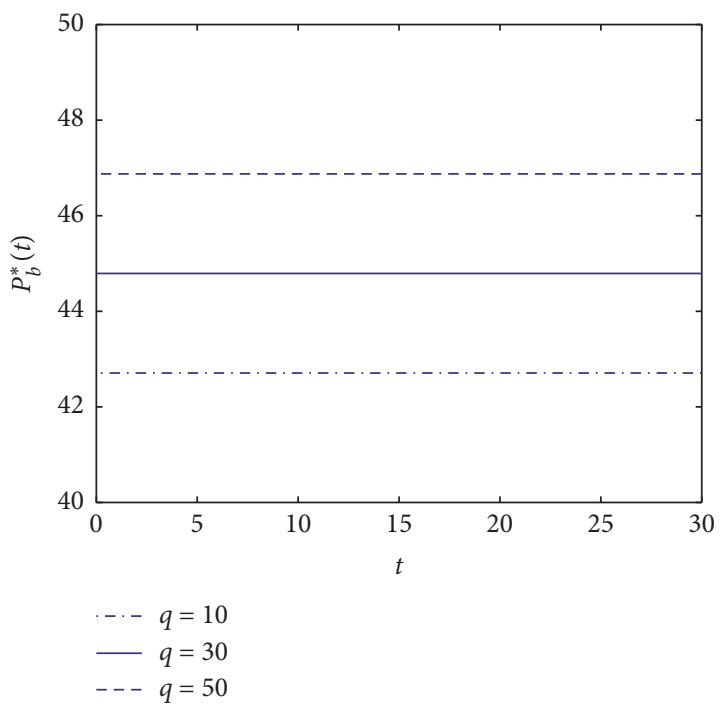

(c)

FIGURE 9: Optimal dynamic prices with different $q$ under different market situations. (a) $a>0$. (b) $a<0$. (c) $a=0$.

TABLE 7: The impact of $q$ on the platform's total transaction volume and total profit under decreasing, increasing, and stable market demand.

\begin{tabular}{|c|c|c|c|c|c|c|}
\hline$q$ & $\psi_{v}^{*}\left(\times 10^{3}\right)$ & $\psi_{b}^{*}\left(\times 10^{3}\right)$ & $\psi_{u}^{*}\left(\times 10^{3}\right)$ & $\pi_{v}^{*}\left(\times 10^{4}\right)$ & $\pi_{b}^{*}\left(\times 10^{4}\right)$ & $\pi_{u}^{*}\left(\times 10^{4}\right)$ \\
\hline 5 & 3.60 & 7.09 & 10.92 & 3.84 & 8.95 & 22.12 \\
\hline 10 & 3.69 & 7.18 & 10.98 & 3.96 & 9.12 & 22.26 \\
\hline 15 & 3.78 & 7.26 & 11.04 & 4.07 & 9.26 & 22.34 \\
\hline 20 & 3.87 & 7.35 & 11.10 & 4.17 & 9.35 & 22.36 \\
\hline 25 & 3.95 & 7.44 & 11.16 & 4.24 & 9.41 & 22.32 \\
\hline 30 & 4.04 & 7.53 & 11.22 & 4.29 & 9.43 & 22.23 \\
\hline 35 & 4.13 & 7.61 & 11.28 & 4.31 & 9.42 & 22.07 \\
\hline 40 & 4.22 & 7.70 & 11.34 & 4.32 & 9.36 & 21.85 \\
\hline 45 & 4.30 & 7.79 & 11.40 & 4.29 & 9.25 & 21.57 \\
\hline 50 & 4.39 & 7.88 & 11.46 & 4.24 & 9.11 & 21.22 \\
\hline
\end{tabular}

decreasing market demand, Figure 10(a) shows that $P_{v}^{*}(0.7$, $t)<P_{v}^{*}(0.5, t)$ when $t<t_{3}, P_{v}^{*}(0.7, t)=P_{v}^{*}(0.5, t)$ when $t=t_{3}$, and $P_{v}^{*}(0.7, t)>P_{v}^{*}(0.5, t)$ when $t>t_{3}$, and the time point $t_{1}$ moves forward when $r$ moves from 0.7 to 0.5 ; the time point $t_{1}$ of $r=0.5$ has actually moved out of $T$ in our simulations. For increasing market demand, as depicted in Proposition 3 (b), Figure 10(b) shows that $P_{u}^{*}(0.7, t)<P_{u}^{*}(0.5, t)$ and the time point $t_{2}$ is pushed backward when $r$ from 0.5 to 0.7 ; this implies the peak congestion period is reduced. For stable market demand, Figure $10(\mathrm{c})$ shows that $P_{b}^{*}(0.7, t)<P_{b}^{*}(0.5$, $t$ ), which is consistent with Proposition 3 (c).

From Table 8, we observe the following:

(i) With decreasing market demand, the platform's total transaction volume increases first and then decreases when $r$ increases. When the total transaction volume is maximized, $r$ is 0.65 . However, under increasing market demand and stable market demand, the total transaction volume increases when $r$ increases.

(ii) For the same $r$, the total transaction volume under increasing market demand is higher than under stable market demand, and the volume under stable market demand is higher than under decreasing market demand. That is, $\psi_{u}^{*}(r)>\psi_{b}^{*}(r)>\psi_{v}^{*}(r)$.

(iii) Regardless of market demand, the platform's total profit increases first and then decreases when $r$ increases. The total profit is the largest when $r=0.35$ under decreasing market demand, that is, $\pi_{v}^{*}\left(r_{v}^{*}=0.35\right) \geqq \pi_{v}^{*}(r)$. The total profit is the largest when $r=0.25$ under stable market demand, that is, $\pi_{b}^{*}\left(r_{b}^{*}=0.25\right) \geqq \pi_{b}^{*}(r)$. The total profit is the largest when $r=0.4$ under increasing market demand, that is, $\pi_{u}^{*}\left(r_{u}^{*}=0.4\right) \geqq \pi_{u}^{*}(r)$. Moreover, we find that when the total profit is maximized, $r$ varies under different market demand situations. That is, $r_{v}^{*}=0.35 \neq r_{b}^{*}=0.25 \neq r_{u}^{*}=0.4$.

(iv) For the same $r$, the total profit under increasing market demand is higher than under stable demand, and the profit under stable market demand is higher than under decreasing market demand. That is, $\pi_{u}^{*}(r)>\pi_{b}^{*}(r)>\pi_{v}^{*}(r)$.

From observation (iii), we note that the platform obviously cannot set a low rate of return for its own profit maximization in reality-that is, $r_{b}^{*}=0.25, r_{v}^{*}=0.35$, or 


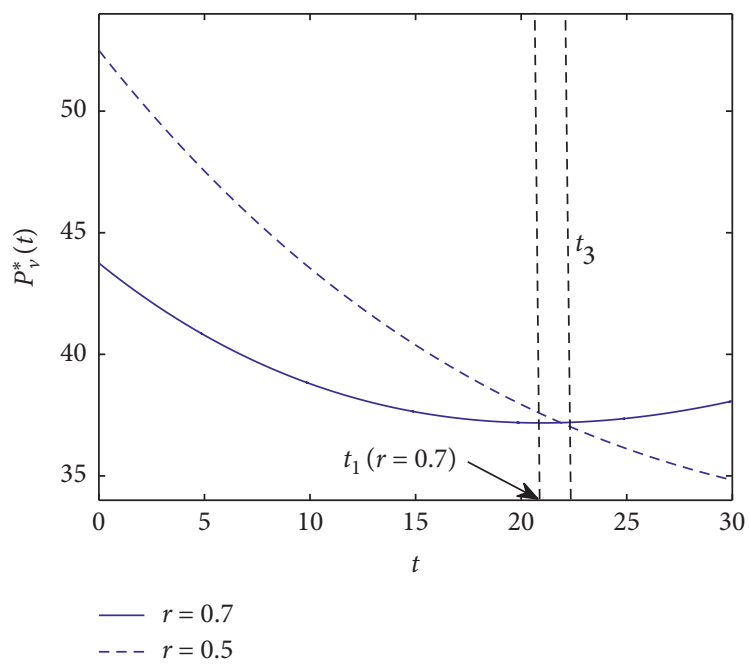

(a)

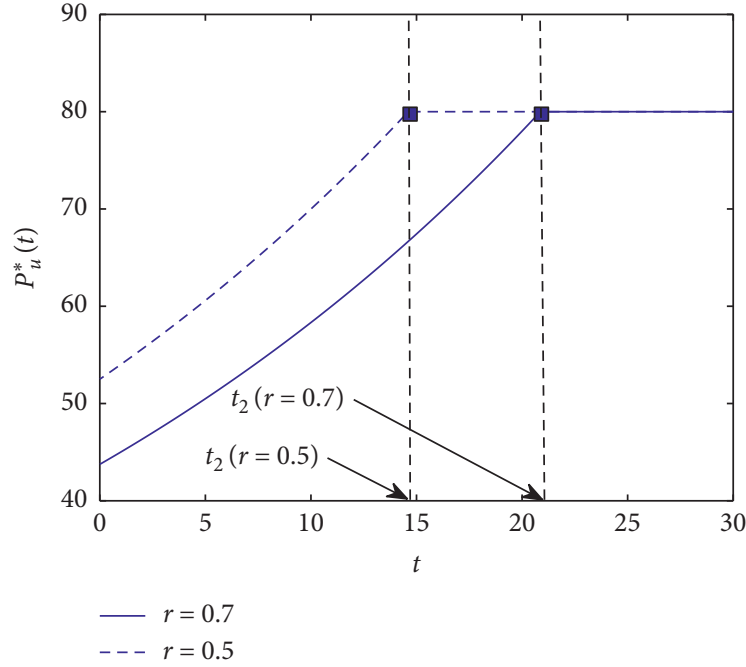

(b)

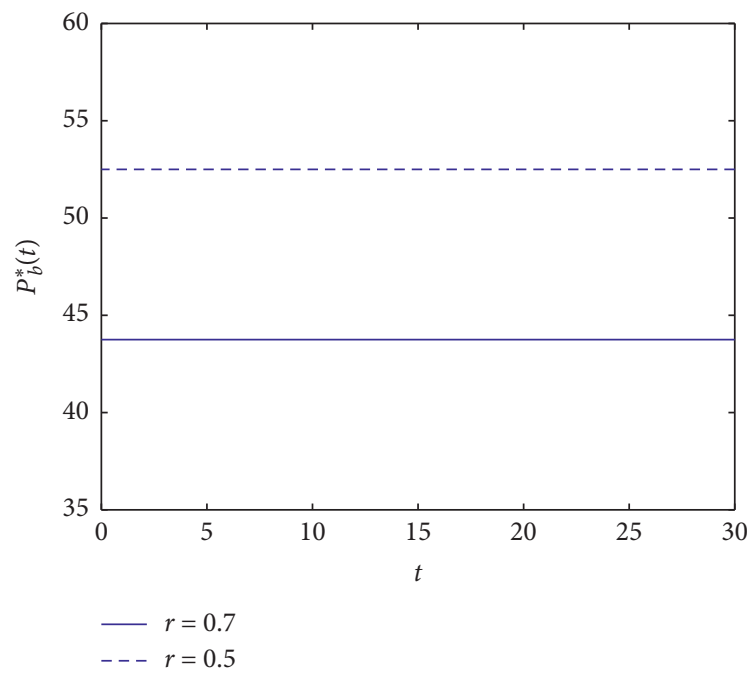

(c)

FIGURE 10: Optimal dynamic prices with different $r$ values under different market situations. (a) $a>0$. (b) $a<0$. (c) $a=0$.

TABLE 8: The impact of $r$ on the platform's total transaction volume and total profit under decreasing, increasing, and stable market demand.

\begin{tabular}{|c|c|c|c|c|c|c|}
\hline$r$ & $\psi_{v}^{*}\left(\times 10^{3}\right)$ & $\psi_{b}^{*}\left(\times 10^{3}\right)$ & $\psi_{u}^{*}\left(\times 10^{3}\right)$ & $\pi_{v}^{*}\left(\times 10^{4}\right)$ & $\pi_{b}^{*}\left(\times 10^{4}\right)$ & $\overline{\pi_{u}^{*}\left(\times 10^{4}\right)}$ \\
\hline 0.2 & 1.24 & 3.60 & 3.83 & 6.46 & 21.46 & 24.15 \\
\hline 0.25 & 1.80 & 4.20 & 4.75 & 7.93 & 21.88 & 27.91 \\
\hline 0.3 & 2.27 & 4.73 & 5.64 & 8.60 & 21.52 & 30.60 \\
\hline 0.35 & 2.67 & 5.19 & 6.48 & 8.73 & 20.62 & 32.26 \\
\hline 0.4 & 3.02 & 5.60 & 7.28 & 8.50 & 19.38 & 32.98 \\
\hline 0.45 & 3.30 & 5.97 & 8.03 & 8.03 & 17.90 & 32.83 \\
\hline 0.5 & 3.54 & 6.30 & 8.74 & 7.41 & 16.29 & 31.91 \\
\hline 0.55 & 3.72 & 6.60 & 9.40 & 6.68 & 14.59 & 30.31 \\
\hline 0.6 & 3.84 & 6.87 & 10.01 & 5.89 & 12.85 & 28.13 \\
\hline 0.65 & 3.90 & 7.12 & 10.58 & 5.05 & 11.09 & 25.45 \\
\hline 0.7 & 3.87 & 7.35 & 11.10 & 4.17 & 9.35 & 22.36 \\
\hline 0.75 & 3.72 & 7.56 & 11.58 & 3.22 & 7.64 & 18.94 \\
\hline
\end{tabular}

$r_{u}^{*}=0.4$-because the platform must consider the basic income of drivers and motivate their participation. In our study, because we do not focus on the platform's incentives for drivers, we find that the optimal $r$ is relatively small. However, this has no impact on our analysis of the relationship between $r$ and the platform's profit. 


\section{Conclusions, Managerial Insights, and Future Research}

This paper examines the dynamic pricing problems of ridehailing platforms under the imbalance between the supply and the demand caused by the market demand fluctuation. Unlike existing demand models, which usually consider the effect of price, we combine the effects of price and time on demand. We model demand as an exponential function of time, introducing the coefficient of demand fluctuation to represent three different market demand situations: decreasing demand, increasing demand, and stable demand. We use optimal control theory to construct our models based on the state change in supply and demand, with the aim of maximizing the platform's total profit in a finite service time.

7.1. Concluding Remarks. Due to the fluctuations of passenger demand, ride-hailing platforms often face undesirable situations of insufficient supply or insufficient demand. Determining how to match passenger supply and demand by optimally setting platform prices is an important issue. In this study, we contribute to the literature on demand function and supply function (e.g., Cachon et al. [8]; Hu and Zhou [4]; Liu et al. [16]). In considering the scenarios of both decreasing and increasing passenger demand, we investigate the dynamic pricing policies of a ride-hailing platform and obtain the optimal pricing strategies under different market demand situations. Some key parameters, such as the coefficient of demand fluctuation, service quality, and fixed commission rate, are examined so we can better understand how they affect pricing decisions. The main conclusions are as follows:

(1) The optimal prices vary dynamically over time in different cases under different market demand situations. For decreasing market demand, with the passage of time, when we do not consider opportunity cost, the optimal price decreases first and then remains unchanged; when we consider the opportunity cost, the optimal price decreases first and then increases. For increasing market demand, with the passage of time, when we do not consider the ceiling price constraint, the optimal price increases; when we consider the ceiling price constraint, the optimal price increases first and then remains unchanged. For stable market demand, the optimal price does not change over time.

(2) The optimal prices dynamically affect the platform's supply capacity and demand. For decreasing market demand, on the one hand, the platform reduces the excess supply capacity through the optimal price to avoid too many drivers accepting bookings; on the other hand, to some extent, the optimal price also alleviates the weakening market demand. For increasing market demand, the platform greatly improves the supply capacity through the optimal price, which can satisfy more people in time; the increase in the optimal price decreases the demand but satisfies passengers who are more anxious for the ride service. For stable market demand, the platform's supply capacity and demand do not change over time under the optimal price.

(3) The platform should adjust its pricing decisions according to different key parameters. (a) In terms of the impact of the coefficient of demand fluctuation, for decreasing market demand, when market demand decreases faster, the optimal price is relatively lower. When the coefficient of demand fluctuation is smaller than the critical value, the time point of the lowest price becomes larger. However, when the coefficient is higher than the critical value, the time point becomes smaller. For decreasing market demand, when market demand increases faster, the optimal price is relatively higher, and thus the time point of the ceiling price is smaller. (b) In terms of the impact of service quality, regardless of market demand, the optimal price should be raised when service quality is improved. When service quality deteriorates, the time point of the lowest price is unchanged but that of the ceiling price is larger. (c) In terms of the impact of the fixed commission rate, regardless of market demand, the optimal price should be raised when the platform reduces the fixed commission rate. When the platform adopts a higher fixed commission rate, the time point of the lowest price is smaller, but that of the ceiling price is larger.

(4) The numerical results show that the key parameters have some effect on the platform's total transaction volume and total profit. (a) In terms of the impact of the coefficient of demand fluctuation, for decreasing market demand, when market demand decreases faster, the total transaction volume and total profit are smaller. For increasing market demand, when market demand increases faster, the total transaction volume and total profit are larger. (b) In terms of the impact of service quality, regardless of market demand, the total transaction volume is larger when service quality is improved and the total profit is concave in service quality. The optimal service quality when the total profit is largest under different market demand scenarios is different. For the same service quality, the total transaction volume and total profit under increasing market demand are higher than under stable market demand, and the volume and profit under stable market demand are higher than under decreasing market demand. (c) In terms of the impact of the fixed commission rate, regardless of market demand, the total transaction volume is larger when the platform adopts a higher fixed commission rate and the total profit is concave in the fixed commission rate. The optimal, profit-maximizing commission rate depends on the market demand (scenario). However, when the platform adopts the same fixed commission rate under different market demand scenarios, the total 
transaction volume and total profit under increasing market demand are higher than under stable market demand, and the volume and profit under stable market demand are higher than under decreasing market demand.

7.2. Managerial Insights. Based on the above conclusions, we offer several important managerial insights:

(1) This paper can guide ride-hailing platform managers in pricing decisions when the platform faces different market demand situations: decreasing demand, increasing demand, and stable demand. For example, Didi Chuxing is considering dynamically adjusting prices based on supply and demand within certain service regions. Even when dynamic pricing is limited, our research can be a reference for a platform's static pricing decisions, for example, in regard to the range of static prices based on different regions' market demand.

(2) Ride-hailing platforms should set different pricing decisions according to the degree of market demand fluctuation in different service regions. For example, Didi Chuxing tends to have more pronounced price changes in some of Shanghai's CBD areas and subway stations.

(3) The fixed commission rate design can affect the platform's pricing decisions. The platform managers should have a flexible commission strategy according to the different service regions and different market demands to thus reduce costs while providing better service and maximizing revenue.

(4) All platform companies should have social responsibility, and a ride-hailing platform is no exception. Some of the ride-hailing platform's social responsibilities are to reduce traffic congestion, make travel better, and help users improve the travel efficiency (e.g., Didi Chuxing and Meituan; Zhong et al. [59]). From this perspective, the platform can change service quality or improve its fixed commission rate to achieve the purpose of improving traffic conditions when market demand is increasing. For example, the platform reduces the service quality by changing the maximum matching distance between the passenger and the driver, which can reduce congestion passengers appropriately and improve the travel efficiency of the remaining passengers through long-distance driver scheduling.

(5) As some new ride-hailing platforms enter the market, they tend to pursue the maximization of transaction volume in the early stages to compete for market share. For example, the Meituan ride-hailing platform, aiming to maximize its transaction volume, competes with Didi Chuxing for market share. Our study might be of interest to these new platforms. Managers can improve the service quality and/or the commission rate to maximize the transaction volume under different market demand situations.
7.3. Future Research. Our work has some limitations and can be extended in several ways. First, one might examine more realistic scenarios with our models. For example, it would be reasonable to consider the drivers' loss avoidance behavior under decreasing market demand or to consider the passengers' booking cancellation behavior under increasing market demand. Also, it would be more applicable in scenarios considering the supply-demand uncertainty and other initial states. Second, for simplicity, we ignore the spatial dimension that is important to a platform's pricing decisions. Future work can use the distance factor to investigate a platform's pricing strategies under market demand fluctuation. Third, future research can analyze the optimal static prices (including surge price) under different market demand situations when dynamic pricing is constrained. It would be interesting to compare the optimal static prices to the optimal dynamic prices. Fourth, future models can introduce the drivers' incentive constraints. For example, it would be valuable to consider the drivers' participation constraint and incentive compatible constraint through the lens of principal-agent theory. Fifth, as more than one platform compete for both demand and supply in reality, future research can examine how two or more platforms compete under the different market demand scenarios of this study.

\section{Appendix}

Proof of Lemma 1. The second partial derivative of $H_{v}$ with respect to $P_{v}(t)$ can be calculated as

$$
\frac{\partial^{2} H_{v}}{\partial P_{v}^{2}}=-2 \beta \cdot(1-r)
$$

Since $\beta>0,0<r<1$, and then by (A.1), we have $\partial^{2} H_{v} / \partial P_{v}^{2}<0$, which indicates that $\pi_{v}\left(P_{v}, t\right)$ is a concave function of $P_{v}(t)$. Obviously, we can get Lemma 1 .

Proof of Theorem 1. Since the supply and demand balance of the ride-hailing platform at time 0 , then by (1) and (2), we know $s(W(0)-\tau)=\alpha-\beta P(0)+\gamma q$.

We obtain

$$
P(0)=\frac{\alpha+\gamma q+s \varepsilon}{s r+\beta} .
$$

By equation (7), the optimal price at time 0 can be obtained:

$$
\begin{aligned}
P_{v}^{*}(0)= & \frac{\alpha}{2 \beta}+\frac{\gamma q+s \varepsilon}{s r+\beta}-\frac{c T(s r+\beta)}{2 \beta(1-r)} \\
& -\frac{\alpha(s r-\beta) \cdot\left(1-e^{-a T}\right)}{2 \beta T a(s r+\beta)}+\frac{v(T)}{T(s r+\beta)} .
\end{aligned}
$$

Next, by (A.2) and (A.3), we can get

$$
v(T)=\frac{c T^{2}(s r+\beta)^{2}}{2 \beta(1-r)}+\frac{\alpha(s r-\beta)\left(1-e^{-a T}\right)}{2 \beta a}+\frac{\alpha T}{2}-\frac{s r \alpha T}{2 \beta} .
$$


Then, by substituting $v(T)$ into (12) and (13), respectively, we can get equations (14) and (15); next substituting equation (14) into (1) and (2), respectively, equations (16) and (17) can be obtained.

Thus, Theorem 1 is proved.

Proof of remark in Theorem 1. By equations (16) and (17) of Theorem 1, the excess supply capacity of the platform at time $t$ can be obtained as follows:

$$
v^{*}(t)=\int_{0}^{t}\left(S_{v}^{*}(t)-D_{v}^{*}(t)\right) \mathrm{d} t .
$$

The first derivative of $v^{*}(t)$ with respect to $t$ can be calculated as

$$
\frac{\mathrm{d} v^{*}(t)}{\mathrm{d} t}=S_{v}^{*}(t)-D_{v}^{*}(t)
$$

Since $v^{*}(t)=0$, in order to satisfy $v^{*}(t) \geqq 0$, we only need $\left(\mathrm{d} v^{*}(t) / \mathrm{d} t\right) \geq 0$, and we use $f(t)=\left(\mathrm{d} v^{*}(t) / \mathrm{d} t\right) \geq 0$; by equations (16) and (17) of Theorem $1, f(t)$ can be obtained as follows:

$$
f(t)=\frac{\alpha(s r-\beta)}{2 \beta} \cdot e^{-a t}+\frac{c(s r+\beta)^{2}}{\beta(1-r)} \cdot t-\frac{\alpha(s r-\beta)}{2 \beta} .
$$

The first derivative of $f(t)$ with respect to $t$ can be calculated as

$$
f^{\prime}(t)=-\frac{a \alpha(s r-\beta)}{2 \beta} \cdot e^{-a t}+\frac{c(s r+\beta)^{2}}{\beta(1-r)} .
$$

Since $f(0)=0$, in order to satisfy $f(t) \geqq 0$, we only need $f^{\prime}(t) \geqq 0$. When $\beta \geqq s r$, obviously, we know $f^{\prime}(t) \geqq 0$ by (A.8). Thus, the constraint $v(t) \geqq 0$ in (9) can be satisfied.

If $\beta<s r, t \geq-(1 / a) \ln \left(2 c(s r+\beta)^{2} / a \alpha(s r-\beta) \cdot(1-r)\right)$ can be obtained by making $f^{\prime}(t) \geqq 0$, if $2 c(s r+\beta)^{2} \geq$ $a \alpha(s r-\beta) \cdot(1-r)$, then $-(1 / a) \ln \left(2 c(s r+\beta)^{2} / a \alpha(s r-\beta)\right.$. $(1-r)) \leq 0$; furthermore, $\quad t \geq 0 \geq-(1 / a) \ln \left(2 c(s r+\beta)^{2} /\right.$ $a \alpha(s r-\beta) \cdot(1-r))$, so $v^{*}(t) \geqq 0$ can be satisfied during the ride service time $[0, T]$. Thus, the constraint $v(t) \geqq 0$ in (9) can be satisfied.

Thus, remark of Theorem 1 is proved.

Proof of Corollary 1. By equation (13) of Theorem 1, the first derivative and second derivative of $P_{v}^{*}(t)$ with respect to $t$ can be calculated, respectively, as follows:

$$
\begin{gathered}
\frac{\mathrm{d} P_{v}^{*}(t)}{\mathrm{d} t}=\frac{-a \alpha}{2 \beta} \cdot e^{-a t}+\frac{c(s r+\beta)}{\beta(1-r)}, \\
\frac{\mathrm{d} P^{2^{*}}{ }_{v}(t)}{\mathrm{d} t^{2}}=\frac{a^{2} \alpha}{2 \beta} \cdot e^{-a t} .
\end{gathered}
$$

Since $\alpha>0, \beta>0$, by (A.10), we know $\mathrm{d} P^{2 *}{ }_{v}(t) / \mathrm{d} t^{2}$, $P_{v}^{*}(t)$ is strictly convex in time $t$.

Thus, Corollary 1 (a) is proved.

For Corollary 1 (b), we assume $c=0$, since $a>0$, by (A.9), we know $\lim _{t \longrightarrow+\infty}\left(\mathrm{d} P_{v}^{*}(t) / \mathrm{d} t\right)=\lim _{t \longrightarrow+\infty}\left((-a \alpha / 2 \beta) \cdot e^{-a t}\right)=0$, there is a threshold $T_{\mathrm{th}}: \underset{T_{t h} \longrightarrow+\infty}{\lim }\left(\mathrm{d} P_{v}^{*}(t) / \mathrm{d} t\right)=$ $\lim _{T_{t h} \longrightarrow+\infty}\left(-(a \alpha / 2 \beta) \cdot e^{-a t}\right)=0$. When $0 \leqq T_{\text {th }} \leqq T$, during the ride service time $\left[0, T_{\mathrm{th}}\right]$, since $\left(\mathrm{d} P_{v}^{*}(t) / \mathrm{d} t\right)<0, P_{v}^{*}(t)$ decreases monotonously, however, during the ride service time $\left[T_{\mathrm{th}}, T\right]$, since $\left(\mathrm{d} P_{v}^{*}(t) / \mathrm{d} t\right)=0, P_{v}^{*}(t)$ stabilizes or remains unchanged. When $T<T_{\mathrm{th}}$, during the ride service time $[0, T]$, since $\left(\mathrm{d} P_{v}^{*}(t) / \mathrm{d} t\right)<0, P_{v}^{*}(t)$ decreases monotonously.

Thus, Corollary 1 (b) is proved.

For Corollary $1(\mathrm{c})$, we assume $c \neq 0, t_{1}$ is obtained from $\left(\mathrm{d} P_{v}^{*}(t) / \mathrm{d} t\right)=0$, i.e., $t_{1}=-(1 / a) \ln (2 c(s r+\beta) / a \alpha(1-r))$; we have known $P_{v}^{*}(t)$ is strictly convex in time $t$ from Corollary 1 (a). When $0 \leqq t_{1} \leqq T$, there exists an extreme point $t_{1}$ and $P_{v}^{\min }=P_{v}^{*}\left(t_{1}\right)$ in the ride service time [0,T], so $P_{v}^{*}(t)$ decreases first and then increases. When $T<t_{1},\left(\mathrm{~d} P_{v}^{*}(t) / \mathrm{d} t\right)<$ $0, P_{v}^{*}(t)$ decreases monotonously in service time $[0, T]$.

Thus, Corollary 1 (c) is proved.

Proof of Corollary 2. By equations (15)-(17) of Theorem 1, we substitute $S_{v}^{*}(t)$ and $D_{v}^{*}(t)$ into (3) and $\psi_{v}^{*}$ can be calculated. Then, we substitute $P_{v}^{*}(t), S_{v}^{*}(t)$, and $D_{v}^{*}(t)$ into (8), and $\pi_{v}^{*}$ can be calculated.

Thus, Corollary 2 is proved.

Proof of Lemma 2. The second partial derivative of $H_{u}$ with respect to $P_{u}(t)$ can be calculated as

$$
\frac{\partial^{2} H_{u}}{\partial P_{u}^{2}}=2 s r \cdot(1-r) .
$$

Since $s>0,0<r<1$, by (A.11), we have $\partial^{2} H_{u} / \partial P_{u}^{2}>0$, which indicates that $\pi_{u}\left(P_{u}, t\right)$ is a convex function of $P_{u}(t)$. Obviously, we can get Lemma 2 .

Proof of Theorem 2. Because of the constraints $u(t) \geqq 0$ and $u(0)=0$ in (20), we only need $u \prime(t) \geq 0$. By (1) and (2), (A.12) can be obtained by making $D(P, t) \geqq S(W, t)$ as follows:

$$
P(t) \leq \frac{\alpha}{\beta+s r} \cdot e^{-a t}+\frac{\gamma q+s \varepsilon}{\beta+s r} .
$$

By (A.12), we know $P_{u}^{\max }$ as follows:

$$
P_{u}^{\max }(t)=\frac{\alpha}{\beta+s r} \cdot e^{-a t}+\frac{\gamma q+s \varepsilon}{\beta+s r} .
$$

Since $\pi_{u}\left(P_{u}, t\right)$ is strictly convex in $P_{u}(t)$ from Lemma 2, $P_{u}^{\max }(t)$ is the optimal price point, i.e., $P_{u}(t)=P_{u}^{\max }(t)$. Next, substituting $P_{u}(t)$ into (1) and (2), respectively, equations (26) and (27) can be obtained:

Thus, Theorem 2 is proved.

Proof of Corollary 3. By equation (25) of Theorem 2, the first derivative and second derivative of $P_{u}(t)$ with respect to $t$ can be calculated, respectively, as follows:

$$
\begin{gathered}
\frac{\mathrm{d} P_{u}^{*}(t)}{\mathrm{d} t}=\frac{-a \alpha}{\beta+s r} \cdot e^{-a t}, \\
\frac{\mathrm{d} P^{2^{*}}{ }_{u}(t)}{\mathrm{d} t^{2}}=\frac{a^{2} \alpha}{\beta+s r} \cdot e^{-a t} .
\end{gathered}
$$


Since $a<0, \alpha>0, \beta>0, s>0,0<r<1$, by (A.14) and (A.15), we know $\left(\mathrm{d} P_{u}^{*}(t) / \mathrm{d} t\right)>0$ and $\left(\mathrm{d} P_{*}^{2 u}(t) / \mathrm{d} t^{2}\right)>0$; obviously, Corollary 3 (a) can be proved.

By equations (26) and (27) of Theorem 2, we know $S_{u}^{*}(t)=D_{u}^{*}(t)$. Also, by $u(0)=0$ and (21), it will be easy to get $u(t)=0$, so order delay cost $h \cdot u(t)=0$. Thus, Corollary 3 (b) is proved.

Proof of Corollary 4. By equations (25)-(27) of Theorem 2, we substitute $S_{u}^{*}(t)$ and $D_{u}^{*}(t)$ into (3), and $\psi_{u}^{*}$ can be calculated. Then, we substitute $P_{u}^{*}(t), S_{u}^{*}(t)$, and $D_{u}^{*}(t)$ into (22), and $\pi_{u}^{*}$ can be calculated.

Thus, Corollary 4 is proved.

Proof of Theorem 3. Since $P_{u}(t) \leqq \bar{P}$, by equation (22) of Theorem 2, we can get $t \leq-(1 / a) \ln (\bar{P} \cdot(s r+\beta)-(\gamma q+s \varepsilon) / \alpha)$, and $t_{2}$ can be obtained as follows:

$$
t_{2}=-\frac{1}{a} \ln \left(\frac{\bar{P} \cdot(s r+\beta)-(\gamma q+s \varepsilon)}{\alpha}\right) .
$$

If $T \leqq t_{2}$, since the platform's service price is not constrained by $\bar{P}$ in the ride service time $[0, T], P_{u}(t)$ is equal to equation (22) of Theorem 2 ; if $0 \leqq t_{2} \leqq T$, when $t \in\left[0, t_{2} P_{u}(t)\right.$ is equal to equation (22) of Theorem 2; however, when $t \in\left(t_{2}\right.$, $T]$, the optimal price will increase to the ceiling price $\bar{P}$ and the platform will maintain $\bar{P}$ so that the platform can use the maximum supply capacity to provide transport.

Thus, Theorem 3 is proved.

Proof of Corollary 5. If $T \leqq t_{2}$, we substitute $P_{u}(t)$ into (1) and (2), respectively, based on Theorem 3, and Corollary 5 (a) can be obtained.

If $0 \leqq t_{2} \leqq T$, similarly, we substitute $P_{u}(t)$ into (1) and (2), respectively; based on Theorem 3, equations (31) and (32) can be obtained. Also, since $S_{u}^{*}(t)=D_{u}^{*}(t)$ when $t \in[0$, $t_{2}$ ] and $S_{u}^{*}(t)=S_{u}^{*}\left(t_{2}\right)=D_{u}^{*}\left(t_{2}\right)<D_{u}^{*}(t)$ when $t \in\left(t_{2}, T\right]$, $S_{u}^{*}(t) \leqq D_{u}^{*}(t)$ if $0 \leqq t_{2} \leqq T$.

Thus, Corollary 5 (b) is proved.

Proof of Corollary 6. If $T \leqq t_{2}$, we substitute $S_{u}^{*}(t)$ and $D_{u}^{*}(t)$ into (3); based on Corollary 5, $\psi_{u}^{*}$ can be calculated, and we substitute $P_{u}^{*}(t), S_{u}^{*}(t)$, and $D_{u}^{*}(t)$ into (22); based on Theorem 3 and Corollary $5, \pi_{u}^{*}$ can be calculated.

Thus, Corollary 6 (a) is proved.

If $0 \leqq t_{2} \leqq T$, similarly, Corollary 6 (b) can be obtained.

Proof of Theorem 4. Because of the supply and demand balance of the ride-hailing platform at time 0 , then by (1) and (2), we know $P(0)=P(0)=(\alpha+\gamma q-s \varepsilon / s r+\beta)$, i.e., (A.2). Also, the supply and demand balance of the ride-hailing platform at time 0 will remain as the market demand is stable; otherwise, the constraint $v(t)=u(t)=0$ of (29) will not be satisfied. Obviously, $P_{b}^{*}(t)=P(0)$. Next, we substitute $P_{b}^{*}(t)$ into (1) and (2), respectively, and equations (31) and (32) can be obtained.

Thus, Theorem 4 is proved.
Proof of Corollary 7. By equations (37)-(39) of Theorem 4, we substitute $S_{b}^{*}(t)$ and $D_{b}^{*}(t)$ into (3), and $\psi_{b}^{*}$ can be calculated. Then, we substitute $P_{b}^{*}(t), S_{b}^{*}(t)$, and $D_{b}^{*}(t)$ into (35), and $\pi_{b}^{*}$ can be calculated.

Thus, Corollary 7 is proved.

Proof of Proposition 1. First, for the market demand decreases (i.e., $a>0$ ), by calculating $\mathrm{d} P_{v}^{*}(t) / \mathrm{d} a$, we find that

$$
\frac{\mathrm{d} P_{v}^{*}(t)}{\mathrm{d} a}=-\frac{\alpha \cdot t}{2 \beta} \cdot e^{-a t} \leq 0 . a .
$$

For the critical point $t_{1}$, we know $P_{v}^{\min }=P_{v}^{*}(t)$; by calculating $\mathrm{d} t_{1} / \mathrm{d} a$, we get

$$
\frac{\mathrm{d} t_{1}}{\mathrm{~d} a}=\frac{1}{a^{2}} \cdot\left(1+\ln \left(\frac{2 c(s r+\beta)}{a \alpha(1-r)}\right)\right) .
$$

The critical point $a^{*}$ can be obtained from $\mathrm{d} t_{1} / \mathrm{d} a=0$, that is,

$$
a^{*}=\frac{2 c e(s r+\beta)}{\alpha(1-r)} \text {. }
$$

Combining equation (A.18) with equation (A.19), it will be easy to get the following: if $a \leqq a^{*}, \mathrm{~d} t_{1} / \mathrm{d} a \geq 0$; if $a>a^{*}$, $\mathrm{d} t_{1} / \mathrm{d} a<0$.

Then, for the market demand increases (i.e., $a<0$ ), when $t \in\left[0, t_{2}\right]$, by calculating $\mathrm{d} P_{u}^{*}(t) / \mathrm{d} a$, we find that

$$
\frac{\mathrm{d} P_{u}^{*}(t)}{\mathrm{d} a}=-\frac{\alpha \cdot t}{\beta+s r} \cdot e^{-a t} \leq 0 .
$$

For the critical point $t_{2}$, we know $P_{u}^{\max }=P_{u}^{*}\left(t_{2}\right)=\bar{P}$; by calculating $\mathrm{d} t_{2} / \mathrm{d} a$, we get

$$
\frac{\mathrm{d} t_{2}}{\mathrm{~d} a}=\frac{1}{a^{2}} \cdot \ln \left(\frac{\bar{P} \cdot(s r+\beta)-(\gamma q+s \varepsilon)}{\alpha}\right)>0 .
$$

Obviously, according to the derivation above, we can get Proposition 1.

Thus, Proposition 1 is proved.

Proof of Proposition 2. By calculating $\mathrm{d} P_{v}^{*}(t) / \mathrm{d} q$ and $\mathrm{d} P_{u}^{*}(t) / \mathrm{d} q$ and when $t \in\left[0, t_{2}\right]$ and $\mathrm{d}_{b}^{*}(t) / \mathrm{d} q$, we find that

$$
\frac{\mathrm{d} P_{v}^{*}(t)}{\mathrm{d} q}=\frac{\mathrm{d} P_{u}^{*}(t)}{\mathrm{d} q}=\frac{\mathrm{d} P_{b}^{*}(t)}{\mathrm{d} q}=\frac{\gamma}{\beta+s r}>0 .
$$

For the critical point $t_{1}$ when market demand decreases, obviously, by calculating $\mathrm{d} t_{1} / \mathrm{d} q$, we get

$$
\frac{\mathrm{d} t_{1}}{\mathrm{~d} q}=0 \text {. }
$$

For the critical point $t_{2}$ when market demand increases, we know $P_{u}^{\max }=P_{u}^{*}\left(t_{2}\right)=\bar{P}$; by calculating $\mathrm{d} t_{2} / \mathrm{d} q$, we get

$$
\frac{\mathrm{d} t_{2}}{\mathrm{~d} q}=\frac{1}{a} \cdot \frac{\alpha \gamma}{\bar{P}(s r+\beta)-(\gamma q+s \varepsilon)}<0 .
$$

Obviously, according to the derivation above, we can get Proposition 2.

Thus, Proposition 2 is proved. 
Proof of Proposition 3. First, for the market demand decreases (i.e., $a>0)$, by calculating $\mathrm{d} P_{v}^{*}(t) / \mathrm{d} r$, we get

$$
\frac{\mathrm{d} P_{v}^{*}(t)}{\mathrm{d} r}=\frac{c(s+\beta) \cdot t}{\beta(1-r)^{2}}-\frac{s(\alpha+\gamma q+s \varepsilon)}{(s r+\beta)^{2}} \text {. }
$$

There exists a critical point $t_{3}$ which can be obtained from $\mathrm{d} P_{v}^{*}(t) / \mathrm{d} r=0$, that is,

$$
t_{3}=\frac{\beta s(\alpha+\gamma q+s \varepsilon) \cdot(1-r)^{2}}{c(s+\beta) \cdot(s r+\beta)^{2}} .
$$

For the critical point $t_{1}$, we know $P_{v}^{\min }=P_{v}^{*}\left(t_{1}\right)$; by calculating $\mathrm{d} t_{1} / \mathrm{d} r$, we get

$$
\frac{\mathrm{d} t_{1}}{\mathrm{~d} r}=-\frac{1}{a} \cdot \frac{s(1-r)+r(s r+\beta)}{(s r+\beta) \cdot(1-r)}<0 .
$$

Second, for the market demand increases (i.e., $a<0$ ), when $t \in\left[0, t_{2}\right]$, by calculating $\mathrm{d} P_{u}^{*}(t) / \mathrm{d} r$, we find that

$$
\frac{\mathrm{d} P_{u}^{*}(t)}{\mathrm{d} r}=-\frac{s \cdot\left(e^{-a t}+\gamma q+s \varepsilon\right)}{(\beta+s r)^{2}}<0 .
$$

For the critical point $t_{2}$, we know $P_{u}^{\max }=P_{u}^{*}\left(t_{2}\right)=\bar{P}$; by calculating $\mathrm{d} t_{2} / \mathrm{d} r$, we get

$$
\frac{\mathrm{d} t_{2}}{\mathrm{~d} r}=-\frac{1}{a} \cdot \frac{\alpha s \bar{P}}{\bar{P}(s r+\beta)-(\gamma q+s \varepsilon)}>0 .
$$

Finally, we analyze the relation of the optimal price with respect to $r$ when the market demand is stable (i.e., $a=0$ ). By calculating $\mathrm{d} P_{b}^{*}(t) / \mathrm{d} r$, we find that

$$
\frac{\mathrm{d} P_{b}^{*}(t)}{\mathrm{d} r}=-\frac{s \cdot(\alpha+\gamma q+s \varepsilon)}{(\beta+s r)^{2}}<0 .
$$

Obviously, according to the derivation above, we can get Proposition 3.

Thus, Proposition 3 is proved.

\section{Data Availability}

The data used to support the findings of this study are available from the corresponding author upon request.

\section{Conflicts of Interest}

The authors declare that there are no conflicts of interest regarding the publication of this article.

\section{Acknowledgments}

This research was partially supported by the National Natural Science Foundation of China (Grant nos. 71572033 and 71832001).

\section{References}

[1] H. Ma, F. Fang, and D. C. Parkes, "Spatio-temporal pricing for ridesharing platforms," 2018, https://arxiv.org/abs/1801. 04015.

[2] Uber, 10 billion trips, 2018, https://www.uber.com/newsroom/ 10-billion.
[3] MarketWatch, Ride-Hailing Industry Expected to Grow Eightfold to \$285 Billion By2030, MarketWatch, New York, NY, USA, 2017, http://www.marketwatch.com/story/ridehailing-industry-expected-to-grow-eightfold-to-285-billionby-2030-2017-05-24.

[4] M. Hu and Y. Zhou, Price, Wage and Fixed Commission in On-Demand matching, Working Paper, University of Toronto, Toronto, Canada, 2019, https://ssrn.com/abstract=2949513.

[5] J. Cramer and A. B. Krueger, "Disruptive change in the taxi business: the case of Uber," American Economic Review, vol. 106, no. 5, pp. 177-182, 2016.

[6] Xinhua News Agency, How Does "Ride-Hailing" Get Better and Better?, Xinhua News Agency, Beijing, China, 2019.

[7] C. W. Yan, H. Zhu, N. Korolko, and D. Woodard, "Dynamic pricing and matching in ride-hailing platforms," Naval Research Logistics, vol. 66, no. 8, pp. 1-20, 2019, https://ssrn.com/ abstract $=3258234$.

[8] G. P. Cachon, K. M. Daniels, and R. Lobel, "The role of surge pricing on a service platform with self-scheduling capacity," Manufacturing \& Service Operations Management, vol. 19, no. 3, pp. 368-384, 2017.

[9] J. Hall, K. Cory, and N. Chris, The Effects of Uber's Surge Pricing: A Case Study, The University of Chicago Booth School of Business, Chicago, IL, USA, 2015, https://www.valuewalk.com/ 2015/09/uber-surge-pricing/?utm_source=mailchimp\&utm_ medium=email\&utm_campaign=EMAIL_DAILY\&utm_ content=quick_link.

[10] P. Afèche, L. Zhe, and M. Costis, "Ride-hailing networks with strategic drivers: the impact of platform control capabilities on performance," University of Toronto, Toronto, Canada, 2018, https://ssrn.com/abstract=3120544.

[11] G. Feng, G. Kong, and Z. Wang, We are on the Way: Analysis of On-Demand Ride-Hailing Systems, University of Minnesota, Minneapolis, Minnesota, 2018, https://ssrn.com/ abstract $=2960991$.

[12] M. Hu and Y. Zhou, Dynamic Type Matching, Rotman School of Management, Toronto, Canada, 2019, https://ssrn.com/ abstract $=2592622$.

[13] L. Sun, R. H. Teunter, M. Z. Babai, and G. Hua, "Optimal pricing for ride-sourcing platforms," European Journal of Operational Research, vol. 278, no. 3, pp. 783-795, 2019.

[14] K. Bimpikis, O. Candogan, and D. Saban, "Spatial pricing in ride-sharing networks," Operations Research, vol. 67, no. 3, pp. 744-769, 2019.

[15] R. Desiraju and S. Moorthy, "Managing a distribution channel under asymmetric information with performance requirements," Management Science, vol. 43, no. 12, pp. 1628-1644, 1997.

[16] W. Liu, X. Yan, W. Wei, and D. Xie, "Pricing decisions for service platform with provider's threshold participating quantity, value-added service and matching ability," Transportation Research Part E: Logistics and Transportation Review, vol. 122, pp. 410-432, 2019.

[17] L. Lu, J. Zhang, and W. Tang, "Optimal dynamic pricing and replenishment policy for perishable items with inventorylevel-dependent demand," International Journal of Systems Science, vol. 47, no. 6, pp. 1480-1494, 2016.

[18] A. Herbon and E. Khmelnitsky, "Optimal dynamic pricing and ordering of a perishable product under additive effects of price and time on demand," European Journal of Operational Research, vol. 260, no. 2, pp. 546-556, 2017.

[19] L. Feng, "Dynamic pricing, quality investment, and replenishment model for perishable items," International 
Transactions in Operational Research, vol. 26, no. 4, pp. 1558-1575, 2019.

[20] J.-C. Rochet and J. Tirole, "Platform competition in two-sided markets," Journal of the European Economic Association, vol. 1, no. 4, pp. 990-1029, 2003.

[21] J. Rochet and J. Tirole, "Two-sided markets: an overview," in Proceedings of the IDEI Working Papers, pp. 1-44, January 2004, http://web.mit.edu/14.271/www/rochet_tirole.pdf.

[22] M. Armstrong, "Competition in two-sided markets," The RAND Journal of Economics, vol. 37, no. 3, pp. 668-691, 2005.

[23] L. Waverman, "Two-sided telecom markets and the unintended consequences of business strategy," Competition Policy International, vol. 3, no. 1, 2007.

[24] G. G. Parker and M. W. van Alstyne, "Two-sided network effects: a theory of information product design," Management Science, vol. 51, no. 10, pp. 1494-1504, 2005.

[25] A. Gaudeul and B. Jullien, "E-commerce, two-sided markets and info-mediation," in Internet and Digital Economics: Principles, Methods and Applications, pp. 268-290, Cambridge University Press, New York, NY, USA, 2007.

[26] S. P. Anderson and J. J. Gabszewicz, "Chapter 18 the media and advertising: a tale of two-sided markets," Handbook of the Economics of Art and Culture, vol. 1, pp. 567-614, 2006.

[27] H. J. Kind and F. Stähler, "Market shares in two-sided media industries," Journal of Institutional and Theoretical Economics, vol. 166, no. 2, pp. 205-211, 2010.

[28] M. Furuhata, M. Dessouky, F. Ordóñez, M.-E. Brunet, X. Wang, and S. Koenig, "Ridesharing: the state-of-the-art and future directions," Transportation Research Part B: Methodological, vol. 57, pp. 28-46, 2013.

[29] X. Wang, F. He, H. Yang, and H. Oliver Gao, "Pricing strategies for a taxi-hailing platform," Transportation Research Part E: Logistics and Transportation Review, vol. 93, pp. 212-231, 2016.

[30] S. Djavadian and J. Y. J. Chow, "An agent-based day-to-day adjustment process for modeling 'Mobility as a Service' with a two-sided flexible transport market," Transportation Research Part B: Methodological, vol. 104, pp. 36-57, 2017.

[31] B. Caillaud and B. Jullien, "Chicken \& egg: competition among intermediation service providers," The RAND Journal of Economics, vol. 34, no. 2, pp. 309-328, 2003.

[32] R. Kolisch and D. Zatta, "Implementation of revenue management in the process industry of north America and Europe," Journal of Revenue and Pricing Management, vol. 11, no. 2, pp. 191-209, 2012.

[33] N. C. Petruzzi and M. Dada, "Pricing and the newsvendor problem: a review with extensions," Operations Research, vol. 47, no. 2, pp. 183-194, 1999.

[34] P. Cao, N. Zhao, and J. Wu, "Dynamic pricing with Bayesian demand learning and reference price effect," European Journal of Operational Research, vol. 279, no. 2, pp. 540-556, 2019.

[35] G. Gallego and G. van Ryzin, "Optimal dynamic pricing of inventories with stochastic demand over finite horizons," Management Science, vol. 40, no. 8, pp. 999-1020, 1994.

[36] G. Gallego and G. van Ryzin, "A multiproduct dynamic pricing problem and its applications to network yield management," Operations Research, vol. 45, no. 1, pp. 24-41, 1997.

[37] S. A. Smith and D. D. Achabal, "Clearance pricing and inventory policies for retail chains," Management Science, vol. 44, no. 3, pp. 285-300, 1998.

[38] D. Besanko and W. L. Winston, "Optimal price skimming by a monopolist facing rational consumers," Management Science, vol. 36, no. 5, pp. 555-567, 1990.
[39] C. K. Anderson and J. G. Wilson, "Wait or buy? the strategic consumer: pricing and profit implications," Journal of the Operational Research Society, vol. 54, no. 3, pp. 299-306, 2003.

[40] Y. Aviv and A. Pazgal, "Optimal pricing of seasonal products in the presence of forward-looking consumers," Manufacturing \& Service Operations Management, vol. 10, no. 3, pp. 339-359, 2008.

[41] Y. Levin, J. Mcgill, and M. Nediak, “Optimal dynamic pricing of perishable items by a monopolist facing strategic consumers," Production and Operations Management, vol. 19, no. 1, pp. 40-60, 2010.

[42] Y. Hu, J. Li, and L. Ran, "Dynamic pricing for airline revenue management under passenger mental accounting," Mathematical Problems in Engineering, vol. 2015, Article ID 836434, 8 pages, 2015.

[43] R. R. Varella, J. Frazão, and A. V. M. Oliveira, "Dynamic pricing and market segmentation responses to low-cost carrier entry," Transportation Research Part E: Logistics and Transportation Review, vol. 98, pp. 151-170, 2017.

[44] A. Ajorlou, A. Jadbabaie, and A. Kakhbod, "Dynamic pricing in social networks: the word-of-mouth effect," Management Science, vol. 64, no. 2, pp. 971-979, 2018.

[45] M. Chen and Z.-L. Chen, "Uncertain about your travel plan? Lock it and decide later: dynamic pricing with a fare-lock option," Transportation Research Part E: Logistics and Transportation Review, vol. 125, pp. 1-26, 2019.

[46] J. Chen, M. Dong, Y. Rong, and L. Yang, "Dynamic pricing for deteriorating products with menu cost," Omega, vol. 75, no. 3, pp. 13-26, 2018.

[47] K. Sato, "Price trends and dynamic pricing in perishable product market consisting of superior and inferior firms," European Journal of Operational Research, vol. 274, no. 1, pp. 214-226, 2019.

[48] L. Zha, Y. Yin, and Y. Du, "Surge pricing and labor supply in the ride-sourcing market," Transportation Research Procedia, vol. 23, pp. 2-21, 2017.

[49] H. Guda and U. Subramanian, "Your Uber is arriving: managing on-demand workers through surge pricing, forecast communication, and worker incentives," Management Science, vol. 65, no. 5, pp. 1949-2443, 2019.

[50] T. Wu, M. Zhang, X. Tian, S. Wang, and G. Hua, "Spatial differentiation and network externality in pricing mechanism of online car hailing platform," International Journal of Production Economics, vol. 219, no. 1, pp. 275-283, 2020.

[51] J. Bai, K. C. So, and C. S. Tang, "Coordinating supply and demand on an on-demand service platform with impatient customers," Manufacturing \& Service Operations Management, vol. 21, no. 3, pp. 479-711, 2019.

[52] T. A. Taylor, "On-demand service platforms," Manufacturing \& Service Operations Management, vol. 20, no. 4, pp. 704-720, 2017.

[53] K. S. Moorthy, "Product and price competition in a duopoly," Marketing Science, vol. 7, no. 2, pp. 141-168, 1998.

[54] M. Motta, "Endogenous quality choice: price vs. quantity competition," The Journal of Industrial Economics, vol. 41, no. 2, pp. 113-131, 1993.

[55] A. Ha, X. Long, and J. Nasiry, "Quality in supply chain encroachment," Manufacturing \& Service Operations Management, vol. 18, no. 2, pp. 280-298, 2016.

[56] R. Maihami and I. N. Kamalabadi, "Joint pricing and inventory control for non-instantaneous deteriorating items with partial backlogging and time and price dependent demand," International Journal of Production Economics, vol. 136, no. 1, pp. 116-122, 2012. 
[57] M. Ghoreishi, G.-W. Weber, and A. Mirzazadeh, "An inventory model for non-instantaneous deteriorating items with partial backlogging, permissible delay in payments, inflationand selling price-dependent demand and customer returns," Annals of Operations Research, vol. 226, no. 1, pp. 221-238, 2015.

[58] S. Jørgensen and P. M. Kort, "Optimal pricing and inventory policies: centralized and decentralized decision making," European Journal of Operational Research, vol. 138, no. 3, pp. 578-600, 2002.

[59] Y. Zhong, Z. Lin, Y.-W. Zhou, T. C. E. Cheng, and X. Lin, "Matching supply and demand on ride-sharing platforms with permanent agents and competition," International Journal of Production Economics, vol. 218, pp. 363-374, 2019. 